\title{
PAIR EXCITATIONS AND THE MEAN FIELD APPROXIMATION OF INTERACTING BOSONS, I
}

\author{
M. GRILLAKIS AND M. MACHEDON
}

\begin{abstract}
In our previous work [20, 21] we introduced a correction to the mean field approximation of interacting Bosons. This correction describes the evolution of pairs of particles that leave the condensate and subsequently evolve on a background formed by the condensate. In 21] we carried out the analysis assuming that the interactions are independent of the number of particles $N$. Here we consider the case of stronger interactions. We offer a new transparent derivation for the evolution of pair excitations. Indeed, we obtain a pair of linear equations describing their evolution. Furthermore, we obtain apriory estimates independent of the number of particles and use these to compare the exact with the approximate dynamics.
\end{abstract}

\section{INTRODUCTION}

The purpose of our present work is to investigate certain aspects of the evolution of a large number of indistinguishable Quantum particles (Bosons) under binary interactions. If we call $\psi\left(t, x_{1}, x_{2} \ldots x_{N}\right)$ the wavefunction describing the $N$ particles with $x_{j} \in \mathbb{R}^{3}$ the coordinates for $j=1,2 \ldots N$, then $\psi$ satisfies an evolution equation of the form

$$
(1 / i) \partial_{t} \psi=\left(H_{1}-N^{-1} V\right) \psi
$$

where $H_{1}$ is a sum of the from $\sum_{j=1}^{N} \Delta_{x_{j}}$. The term $V$ models two body interactions of the following general type

$$
V:=(1 / 2) \sum_{x_{j} \neq x_{k}} N^{3 \beta} v\left(N^{\beta}\left(x_{j}-x_{k}\right)\right) \quad ; \quad 0 \leq \beta \leq 1
$$

where $v \in C_{0}^{1}$ is non-negative, spherically symmetric, and decreasing.

In the equation (11) above we consider non-relativistic particles and set $h=2 m=1$ for simplicity. Here and for the rest of this paper we denote $v_{N}:=N^{3 \beta} v\left(N^{\beta} \cdot\right)$. The fact that we consider Bosons means that the wavefunction is invariant under all permutations of the indices $j=1,2 \ldots N$ and one would like to solve the evolution equation under some initial condition at, say $t=0, \psi\left(0, x_{1}, x_{2} \ldots x_{n}\right):=$ 
$\psi_{0}\left(x_{1}, x_{2} \ldots x_{N}\right)$. Presently we are interested in the evolution of factorized (or approximately factorized) initial data i.e. we would like to consider special initial data of the form

$$
\psi_{0}:=\prod_{j=1}^{N} \phi_{0}\left(x_{j}\right) .
$$

The evolution of (11) with initial data (3) is quite complicated for $N$ large and one would like to have an effective approximate description of the evolution. The motivation for this type of problem comes from Bose-Einstein condensation where one considers a large number of identical (indistinguishable) particles in a trap. Einstein following ideas of Bose, observed that nonineracting particles in a box undergo a phase transition at a critical temperature proportional to density ${ }^{2 / 3}$, so that below this temperature a macroscopic number of particles occupy the ground state, furthermore at zero temperature all particles condense to the ground state. It is natural and more realistic to consider interacting particles. Following ideas of Landau a heuristic theory based on the idea of the mean-field approximation was developed by Gross and Pitaevski [32], [22]. On a more fundamental level, the problem of a weakly interacting Quantum gas was taken up in the pioneering work of Lee, Huang and Yang as well as Dyson) [27], [10].

More recent theoretical developments are due to Lieb, Solovej, Yngvanson, Seiringer et. al. see [29] and references therein. In particular, Theorems 6.1, 7.1 in [29], as well as Appendix C in [13] strongly suggest that that the ground state is well approximated by a tensor product (3) where $\phi_{0}$ describes a mean field approximation.

Let us point out that we can in fact treat more general initial conditions corresponding to the $N$ th component of $e^{\sqrt{N} A(\phi)} e^{B(k)}|0\rangle$, see section (2).

Experimental confirmation of Bose-Einstein condensates was finally achieved [1], using alkali atoms. The reason for the use of alkali atoms is the fact that they contain a single valence electron in the outermost $s$-orbital (for example 5 - $s$ for Rubidium). The other contributions to the total spin comes from the nuclear spin. If the nuclear isotope is one with odd number of protons and neutrons it will have a net half integer spin. For example Rubidium 87 has $S=3 / 2$ from the nucleus. The total spin takes the values $S=1$ or $S=2$. If we prepare the sample so that only one of these states is present then this will be a gas of identical Bosons. If two different states are present then we should consider it as a mixture of two different gasses. Since alkali atoms are complicated composite particles their interactions are not known explicitly, which 
means that the potential $v$ in (2) is not explicitly known, moreover one can treat the atoms as Bosons only for a sufficiently dilute gas. At shorter distances the internal structure of the atoms should be taken into account. Here we consider a sufficiently dilute Boson gas and we make the reasonable assumption that the interactions are repulsive i.e. $v \geq 0$ and that they are short range in the sense that $\int v(x) d x<\infty$. It is clear from the above comments that one should consider particles with spin. The present framework can be generalized in this case in a straightforward manner, however in the name of simplicity we forgo this generalization.

Let us comment on the scaling present in the form of binary interactions. The parameter $\beta$ describes the strength of particle interactions. It is a reasonable (but not obvious) idea to assume that the evolution of (1) is approximated by a tensor product i.e.

$$
\psi(t) \approx \prod_{j=1}^{N} \phi\left(t, x_{j}\right)
$$

and the issues are, first to explain the nature of the approximation described in (4) and second to derive an evolution equation for $\phi(t, x)$ consistent with the dynamics of (11). On the second question, the general idea is that the evolution of the mean field $\phi(t, x)$ satisfies an equation of the form

$$
i \partial_{t} \phi=\Delta \phi-g_{\beta}\left(|\phi|^{2}\right) \phi,
$$

and the nonlinear term $g_{\beta}\left(|\phi|^{2}\right)$ depends on $\beta$ in the following manner,

$$
\begin{aligned}
& g_{0}\left(|\phi|^{2}\right)=\int d y\left\{v(x-y)|\phi(t, y)|^{2}\right\} \\
& g_{\beta}\left(|\phi|^{2}\right)=\left(\int v(y) d y\right)|\phi(t, x)|^{2} ; 0<\beta<1 \\
& g_{1}\left(|\phi|^{2}\right)=8 \pi a|\phi(t, x)|^{2},
\end{aligned}
$$

where $a$ appearing in $g_{1}$ is the scattering length corresponding to the potential $v$. In the case $\beta=0$ one obtains a Hartree type evolution for the mean field and considerations similar to our present work where taken up in [20], [21]. The case $\beta=1$ is probably the most interesting. In this case the scaling is critical in the sense that particles develop short scale correlations which in the limit $N \rightarrow \infty$ lead to the appearance of the scattering length in the equation. A heuristic argument for this is well known in the Physics community, however the explanation on how the scattering length emerges from the $N$ body dynamics was recently given in the work of Erdös, Schlein and Yau [16], [15]. 
Our aim is to introduce pair excitations as a correction to the mean field approximation. This goal is achieved by introducing a kernel $k(t, x, y)$ which describes pair excitations and one would like to derive an evolution equation for $k$ consistent with the $N$ body dynamics, which means that we should be able to obtain estimates comparing the exact with the approximate dynamics. The general idea of the approximation can be described in the following manner. Two particles leave the condensate and form a pair $v_{N}\left(x_{1}-x_{2}\right) \phi\left(x_{1}\right) \phi\left(x_{2}\right)$ which in turn drives the evolution of pair interactions. It turns out that a natural way to introduce pair excitations as a correction to the mean field is via a Fock space formalism which we will outline in the next section. Let us comment here on the nature of our approximation. The mean field approximation (4) is a simple description of the $N$-body wavefunction, however the nature of the approximation is quite involved and uses the BBGKY hierarchy and its limit as $N \rightarrow \infty$ as shown by Elgart, Erdös, Schlein and Yau [11, 12, 13, 14, 15, 16]. See the approach of [25], [26], [5] based on space-time estimates. We also mention the related case of 3 body interactions [6], and switchable quadratic traps [7, 8].

Moreover the approximation does not track the exact dynamics, rather its true usefullness lies in the fact that it can (approximately) track observables. In contrast our approximation is more complicated but it tracks the exact dynamics in Fock space norm. As a matter of fact a heuristic explanation of our approximation runs as follows: The $N$-body wave function consists of three parts, particles that live in the condensate, bound pairs and particles that decayed after forming pairs. Controlling the number of particles that formed pairs leads to another justification of the mean field approximation. We will not pursue this line of inquiry here, however the approximation can be readily used to estimate observables. There are two main points in our present work. First we have a new transparent derivation of the evolution equation of pair excitations, indeed we derive a new system of linear equations. Second we obtain apriory estimates for the pair excitations kernel which are independent of $N$ and this, in turn, allows us to estimate the difference between the exact and approximate solutions provided that $\beta$ is sufficiently small $\left(\beta<\frac{1}{6}\right)$.

Our work was inspired by [35] as well as [37]. Previous works directly related to the present are [18] and [24]. See also [2] and 31.

See Theorem (2.3) below for the precise statement of our main result.

The paper is organized as follows. In section 2 we develop the Fock space formalism which is necessary for our computations and derive the evolution equations for the pair excitation kernel. In section 2 
and 3 we we derive the apriory estimates for the mean-field and for the pair excitation kernel. In section 4 we show how this information can be implemented in order to compare the exact solution to our approximation.

\section{FoCK SPACE FORMALISM AND THE NEW DERIVATION}

In this section we introduce the Fock space formalism and the Hamiltonian evolution in symmetric Fock space. $\mathbb{F}$ is a Hilbert space consisting of vectors of the form

$$
|\psi\rangle=\left(\psi_{0}, \psi_{1}\left(x_{1}\right), \psi_{2}\left(x_{1}, x_{2}\right), \ldots\right)
$$

where $\psi_{0} \in \mathbb{C}$ and $\psi_{k}$ are symmetric $L^{2}$ functions. The norm of such a vector is,

$$
\||\psi\rangle\left\|^{2}=\langle\psi \mid \psi\rangle=\left|\psi_{0}\right|^{2}+\sum_{n=1}^{\infty}\right\| \psi_{n} \|_{L^{2}}^{2} .
$$

Thus $\mathbb{F}$ is a direct sum of sectors $\mathbb{F}_{n}$ of the form,

$$
\mathbb{F}=\sum_{n=0}^{\infty} \mathbb{F}_{n} \quad ; \quad \mathbb{F}_{n}:=L_{s}^{2}\left(\mathbb{R}^{3 n}\right)
$$

with $\mathbb{F}_{0}=\mathbb{C}$ and $L_{s}^{2}\left(\mathbb{R}^{3}\right)$ denoting the subspace of symmetric functions. In the Fock space $\mathbb{F}$ we introduce creation and anihilation distribution valued operators denoted by $a_{x}^{*}$ and $a_{x}$ respectively which act on sectors $\mathbb{F}_{n-1}$ and $\mathbb{F}_{n+1}$ in the following manner,

$$
\begin{aligned}
& a_{x}^{*}\left(\psi_{n-1}\right):=\frac{1}{\sqrt{n}} \sum_{j=1}^{n} \delta\left(x-x_{j}\right) \psi_{n-1}\left(x_{1}, \ldots, x_{j-1}, x_{j+1}, \ldots, x_{n}\right) \\
& a_{x}\left(\psi_{n+1}\right):=\sqrt{n+1} \psi_{n+1}\left([x], x_{1}, \ldots, x_{n}\right)
\end{aligned}
$$

with $[x]$ indicating that the variable $x$ is frozen. In addition $a_{x}$ kills $\mathbb{F}_{0}$ i.e. $a_{x}\left(\psi_{0}\right)=0$. The vacuum state will play an important role later and we define it as follows

$$
|0\rangle:=(1,0,0 \ldots)
$$

so that $a_{x}|0\rangle=0$. One can easily check that $\left[a_{x}, a_{y}^{*}\right]=\delta(x-y)$ and since the creation and anihilation operators are distribution valued we can form operators that act on $\mathbb{F}$ by introducing a field, say $\phi(x)$, and form

$$
a_{\bar{\phi}}:=\int d x\left\{\bar{\phi}(x) a_{x}\right\} \quad \text { and } \quad a_{\phi}^{*}:=\int d x\left\{\phi(x) a_{x}^{*}\right\}
$$

where by convention we associate $a$ with $\bar{\phi}$ and $a^{*}$ with $\phi$. These operators are well defined, unbounded, on $\mathbb{F}$ provided that $\phi$ is square 
integrable. The creation and anihilation operators provide a way to introduce coherent states in $\mathbb{F}$ in the following manner, first define

$$
\mathcal{A}(\phi):=\int d x\left\{\bar{\phi}(x) a_{x}-\phi(x) a_{x}^{*}\right\}
$$

and then introduce $N$-particle coherent states as

$$
|\psi(\phi)\rangle:=e^{-\sqrt{N} \mathcal{A}(\phi)}|0\rangle
$$

It is easy to check that

$$
e^{-\sqrt{N} \mathcal{A}(\phi)}|0\rangle=\left(\ldots c_{n} \prod_{j=1}^{n} \phi\left(x_{j}\right) \ldots\right) \quad \text { with } \quad c_{n}=\left(e^{-N} N^{n} / n !\right)^{1 / 2} .
$$

In particular, by Stirling's formula, the main term that we are interested in has the coefficient

$$
c_{N} \approx(2 \pi N)^{-1 / 4}
$$

Thus a coherent state introduces a tensor product in the sector $\mathbb{F}_{N}$, hence we can use such states as a mean field approximation to the Hamiltonian evolution in Fock space, see (41).

The Fock Hamiltonian (acting on Fock space vectors) is

$$
\begin{aligned}
\mathcal{H} & :=\mathcal{H}_{1}-N^{-1} \mathcal{V} \quad \text { where, } \\
\mathcal{H}_{1} & :=\int d x d y\left\{\Delta_{x} \delta(x-y) a_{x}^{*} a_{y}\right\} \quad \text { and } \\
\mathcal{V} & :=\frac{1}{2} \int d x d y\left\{v_{N}(x-y) a_{x}^{*} a_{y}^{*} a_{x} a_{x}\right\}
\end{aligned}
$$

where we set

$$
v_{N}(x-y):=N^{3 \beta} v\left(N^{\beta}|x-y|\right),
$$

and the evolution in Fock space is described by the equation,

$$
\frac{1}{i} \partial_{t}|\psi\rangle=\mathcal{H}|\psi\rangle
$$

which has the formal solution

$$
|\psi(t)\rangle=e^{i t \mathcal{H}}\left|\psi_{0}\right\rangle
$$

Notice that $\mathcal{H}$ preserves the sectors $\mathbb{F}_{n}$ and that $\mathcal{H}$ agrees with the classical Hamiltonian (11) on $\mathbb{F}_{N}$. However in this framework we allow any number of particles to evolve and one is interested, in particular, in the evolution on the sector $\mathbb{F}_{N}$. 
Our goal is to approximate $|\psi(t)\rangle$ in (11) and for this purpose we introduce two fields $\phi(t, x)$ and $k(t, x, y)$ and the associated operators,

$$
\begin{aligned}
\mathcal{A}(\phi) & :=\int d x\left\{\bar{\phi}(t, x) a_{x}-\phi(t, x) a_{x}^{*}\right\} \\
\mathcal{B}(k) & :=\int d x d y\left\{\bar{k}(t, x, y) a_{x} a_{y}-k(t, x, y) a_{x}^{*} a_{y}^{*}\right\} .
\end{aligned}
$$

The coherent initial data are introduced via $\left|\psi_{0}\right\rangle=e^{-\sqrt{N} \mathcal{A}\left(\phi_{0}\right)}|0\rangle$ which means that the initial data are a tensor product on $\mathbb{F}_{N}$ as desired, see (33). Our approximation scheme is

$$
\left|\psi_{\text {appr }}\right\rangle:=e^{-\sqrt{N} \mathcal{A}(t)} e^{-\mathcal{B}(t)} e^{i N \chi(t)}|0\rangle
$$

with $\chi(t)$ a phase factor, and we plan to show that $|\psi(t)\rangle \approx\left|\psi_{\text {appr }}(t)\right\rangle$.

The issue for us is to determine the dynamics of the fields $\phi$ and $k$. It turns out the the evolution of $k$ is described via a set of new fields,

$$
\begin{aligned}
& \operatorname{sh}(k):=k+\frac{1}{3 !} k \circ \bar{k} \circ k+\ldots, \\
& \operatorname{ch}(k):=\delta(x-y)+\frac{1}{2 !} \bar{k} \circ k+\ldots,
\end{aligned}
$$

where $\circ$ indicates composition, namely $k \circ l$ stands for the product,

$$
(k \circ l)\left(x_{1} x_{2}\right):=\int d y\left\{k\left(x_{1}, y\right) l\left(y, x_{2}\right)\right\} .
$$

A crucial property of the above multiplication is that it is not commutative i.e. $k \circ l \neq l \circ k$. In order to describe the evolution we need

$$
\begin{aligned}
g_{N}(t, x, y) & :=-\Delta_{x} \delta(x-y)+\left(v_{N} *|\phi|^{2}\right)(t, x) \delta(x-y) \\
& +v_{N}(x-y) \bar{\phi}(t, x) \phi(t, y) \\
m_{N}(x, y): & =-v_{N}(x-y) \phi(x) \phi(y) .
\end{aligned}
$$

Using $g_{N}$ we can construct two operators as follows: For a function $s(t, x, y)$ symmetric in $(x, y)$ and a function $p(t, x, y)$ conjugate symmetric in $(x, y)$ i.e. $\bar{p}^{T}=p$, we define

$$
\begin{aligned}
\mathbf{S}(s) & :=\frac{1}{i} s_{t}+g_{N}^{T} \circ s+s \circ g_{N} \\
\mathbf{W}(p) & :=\frac{1}{i} p_{t}+\left[g_{N}^{T}, p\right] .
\end{aligned}
$$


The dynamics of the fields are determined via,

$$
\begin{aligned}
& \frac{1}{i} \partial_{t} \phi-\Delta \phi+\left(v_{N} *|\phi|^{2}\right) \phi=0 \\
& \mathbf{S}(\operatorname{sh}(2 k))=m_{N} \circ \operatorname{ch}(2 k)+\overline{\operatorname{ch}(2 k)} \circ m_{N} \\
& \mathbf{W}(\overline{\operatorname{ch}(2 k)})=m_{N} \circ \overline{\operatorname{sh}(2 k)}-\operatorname{sh}(2 k) \circ \bar{m}_{N} .
\end{aligned}
$$

Recall we assume $v \in C_{0}^{1}$ is non-negative, spherically symmetric, and decreasing.

Remark 2.1. It is clear that $\operatorname{ch}(2 k)$ and $\operatorname{sh}(2 k)$ are not independent of each other, thus we can ignore the third equation, however in the form stated above the equations are readily amenable to the derivation of apriori estimates. The equation for $\phi$ is of Hartree type and its formal limit as $N \rightarrow \infty$ is NLS.

The theorem concerning the evolution of the mean field $\phi$ and the pair excitation kernel $k$ reads as follows.

Theorem 2.2. Suppose that $0<\beta<1$ in (9). Given initial data $\phi(0, x):=\phi_{0}(x) \in W^{k, 1}$ ( $k$ derivatives in $L^{1}$, with $k$ sufficiently large) and $k(0, x, y):=0$ the system (17a) (17b) (17c) has global solutions in time which satisfy the apriori estimates,

$$
\begin{aligned}
& \|\phi(t)\|_{H^{s}\left(\mathbb{R}^{3}\right)} \leq C_{s} \\
& \|\phi(t)\|_{L^{\infty}\left(\mathbb{R}^{3}\right)}+\left\|\partial_{t} \phi(t)\right\|_{L^{\infty}\left(\mathbb{R}^{3}\right)} \leq \frac{C}{t^{3 / 2}} \\
& \|\operatorname{sh}(2 k)(t)\|_{L^{2}\left(\mathbb{R}^{6}\right)}+\|\operatorname{ch}(2 k)(t)-\delta\|_{L^{2}\left(\mathbb{R}^{6}\right)} \leq C \log (1+t) .
\end{aligned}
$$

The main difficulty in obtaining the estimates in the theorem above is the fact that $v_{N}$ defined in (9) has a formal limit $v_{N}(x-y) \rightarrow c \delta(x-y)$ which means that $m_{N}$ has a limit which is not square integrable, as a matter of fact it does not belong to any $L^{p}$ for $p>1$. In view of the theorem above, we can compare the exact with the approximate evolutions and the result is the following theorem.

Theorem 2.3. Suppose that $|\psi(t)\rangle$ is the solution of (10) with initial data $\left|\psi_{0}\right\rangle:=e^{-\sqrt{N} \mathcal{A}\left(\phi_{0}\right)}|0\rangle$ and $\left|\psi_{\text {appr }}(t)\right\rangle$ is the approximation in (13) where the evolution of the fields $\phi$ and $k$ is determined from theorem (2.2). Under these conditions the following estimate holds,

$$
\||\psi(t)\rangle-\left|\psi_{\text {appr }}(t)\right\rangle \|_{\mathbb{F}} \leq \frac{C(1+t) \log ^{4}(1+t)}{N^{(1-3 \beta) / 2}} .
$$

provided $0<\beta<\frac{1}{3}$. This is a meaningful approximation of the $N$ th component of $|\psi\rangle$ provided $0<\beta<\frac{1}{6}$, because of formula (7). A slightly 
more precise form of the estimate could be obtained by integrating the right hand side of the inequalities in Proposition (6.1).

Remark 2.4. The real phase factor $\chi$ is described via $\chi(t):=\int^{t} d t_{1}\left\{\mu_{0}\left(t_{1}\right)+\right.$ $\left.N^{-1} \mu_{1}\left(t_{1}\right)\right\}$ where

$$
\mu_{0}(t)=\frac{1}{2} \int d x d y\left\{v_{N}(x-y)|\phi(t, x)|^{2}|\phi(t, y)|^{2}\right\}
$$

and $\mu_{1}$ is a complicated integral given in (73)

Proof. Here is an outline of the proof of this theorem. In order to relate the exact with the approximate solution we introduce the reduced dynamics

$$
\left|\psi_{\text {red }}(t)\right\rangle:=e^{\mathcal{B}(t)} e^{\sqrt{N} \mathcal{A}(t)} e^{i t \mathcal{H}} e^{-\sqrt{N} \mathcal{A}(0)}|0\rangle
$$

i.e. we follow the exact dynamics for time $t$ and then go back following the approximate evolution. Notice that $\left|\psi_{\text {red }}(0)\right\rangle=|0\rangle$ and if our approximation was following the exact evolution we would have that $\left|\psi_{\text {red }}(t)\right\rangle=|0\rangle$. Thus our goal is to estimate the deviation of the evolution from the vacuum state. It is straightforward to compute the evolution of $\left|\psi_{\text {red }}\right\rangle$ and it is

$$
\frac{1}{i} \partial_{t}\left|\psi_{\text {red }}\right\rangle=\mathcal{H}_{\text {red }}\left|\psi_{\text {red }}\right\rangle
$$

where the (self-adjoint) reduced Hamiltonian is,

$$
\begin{aligned}
\mathcal{H}_{\text {red }} & :=\frac{1}{i}\left(\partial_{t} e^{\mathcal{B}}\right) e^{-\mathcal{B}} \\
& +e^{\mathcal{B}}\left(\frac{1}{i}\left(\partial_{t} e^{\sqrt{N} \mathcal{A}}\right) e^{-\sqrt{N} \mathcal{A}}+e^{\sqrt{N} \mathcal{A}} \mathcal{H} e^{-\sqrt{N} \mathcal{A}}\right) e^{-\mathcal{B}} .
\end{aligned}
$$

The main idea is that the evolution of the fields $\phi$ and $k$ is chosen so that the reduced Hamiltonian looks like

$$
\mathcal{H}_{\text {red }}=N \mu(t)+\int d x d y\left\{L(t, x, y) a_{x}^{*} a_{y}\right\}-N^{-1 / 2} \mathcal{E}(t)
$$

where $\mathcal{E}(t)$ is an error term containing polynomials in $\left(a, a^{*}\right)$ up to degree four, and $L$ is some self-adjoint expression which is irrelevant for the rest of the argument.

Next consider

$$
|\widetilde{\psi}\rangle:=e^{-i N \chi(t)}\left(\left|\psi_{\text {red }}\right\rangle\right)-|0\rangle \quad \text { where } \quad \chi(t):=\int^{t} \mu\left(t_{1}\right) d t_{1}
$$

where we called $\mu:=\mu_{0}+N^{-1} \mu_{1}$. Thus

$$
\left(\frac{1}{i} \partial_{t}-\mathcal{H}_{\text {red }}+N \mu(t)\right) e^{-i N \chi(t)}\left|\psi_{\text {red }}\right\rangle=0 .
$$


and therefore

$$
\left(\frac{1}{i} \partial_{t}-\mathcal{H}_{r e d}+N \mu(t)\right)|\widetilde{\psi}\rangle=N^{-1 / 2} \mathcal{E}(t)|0\rangle
$$

The equation above has a forcing term namely $N^{-1 / 2} \mathcal{E}(t)|0\rangle$ and a standard energy estimate together with the fact that $e^{\sqrt{N} \mathcal{A}}$ and $e^{\mathcal{B}}$ are unitary, gives

$$
\begin{aligned}
& \||\psi(t)\rangle-\left|\psi_{\text {appr }}(t)\right\rangle \|_{\mathbb{F}} \\
& =\||\widetilde{\psi}(t)\rangle\left\|_{\mathbb{F}} \leq N^{-1 / 2} \int_{0}^{t} d t_{1}\left\{\| \mathcal{E}\left(t_{1}\right)|0\rangle \|_{\mathbb{F}}\right\} .\right.
\end{aligned}
$$

The proof will be complete if we estimate the right hand side in the above inequality. Notice that $\mathcal{E}|0\rangle$ has entry only in Fock sectors $\mathbb{F}_{j}$ for $j=1,2,3,4$ and in order to estimate it we need the lemma below

Lemma 2.5. The error term is described as follows,

$$
\mathcal{E}:=e^{\mathcal{B}}\left(\mathcal{P}_{3}+N^{-1 / 2} \mathcal{P}_{4}\right) e^{-\mathcal{B}}
$$

where $\mathcal{P}_{3}$ and $\mathcal{P}_{4}$ are cubic and quartic polynomials in $\left(a, a^{*}\right)$ respectively. Moreover the following estimate holds if $0 \leq \beta<\frac{1}{3}$,

$$
\| \mathcal{E}(t)|0\rangle \|_{\mathbb{F}} \leq C N^{3 \beta / 2} \log ^{4}(1+t) .
$$

A more precise estimate is given in Proposition (6.1).

Remark 2.6. The polynomials $\mathcal{P}_{3}$ and $\mathcal{P}_{4}$ appearing in the error term are given by the expressions,

$$
\begin{aligned}
& \mathcal{P}_{3}:=\int d x d y\left\{v_{N}(x-y)\left(\phi(y) a_{x}^{*} a_{y}^{*} a_{x}+\bar{\phi}(y) a_{x}^{*} a_{x} a_{y}\right)\right\} \\
& \mathcal{P}_{4}:=(1 / 2) \int d x d y\left\{v_{N}(x-y) a_{x}^{*} a_{y}^{*} a_{x} a_{y}\right\}
\end{aligned}
$$

as we will see shortly.

The rest of this section is devoted to the derivation of (17a) (17b) (17c). We have to compute $\mathcal{H}_{\text {red }}$ above, see (23), and for this task there are two crucial ingredients. They are based on the formal identities below for any two operators, say $\mathcal{A}$ and $\mathcal{H}$,

$$
\begin{aligned}
e^{\mathcal{A}} \mathcal{H} e^{-\mathcal{A}} & =\sum_{n=0}^{\infty} \frac{1}{n !}\left(\operatorname{ad}_{\mathcal{A}}\right)^{n}(\mathcal{H}) \\
\left(\partial_{t} e^{\mathcal{A}}\right) e^{-\mathcal{A}} & =\sum_{n=1}^{\infty} \frac{1}{n !}\left(\operatorname{ad}_{\mathcal{A}}\right)^{n-1}\left(\mathcal{A}_{t}\right)
\end{aligned}
$$


where $\operatorname{ad}_{\mathcal{A}}(\mathcal{H}):=[\mathcal{A}, \mathcal{H}]$. They indicate that we have to compute repeated commutators of various operators. The series defining the exponentials in (26a), (26b) converge absolutely on the dense subset of vectors with finitely many nonzero entries provided that $\mathcal{A}=\mathcal{A}(\phi)$ is a polynomial of degree one with $\phi \in L^{2}$ or $\mathcal{A}=\mathcal{B}(k)$ is second order with $\|k\|_{L^{2}}$ small. If $\mathcal{B}$ is skew-Hermitian, $e^{\mathcal{B}}$ extends as a unitary operator for all $k \in L^{2}$. This construction is closely related to the Segal-ShaleWeil representation, as explained in [24, [17], 36], and our appendix (17). This calculation was also used in our previous papers [20, 21].

The first observation is the fact that since $\mathcal{A}(\phi)$ is a degree one polynomial, if we denote by $\mathcal{P}_{n}$ a homogeneous polynomial of degree $n$ then commuting with $\mathcal{A}$ produces $\left[\mathcal{A}, \mathcal{P}_{n}\right]=\mathcal{P}_{n-1}$ i.e. a homogeneous polynomial of degree $n-1$. This in turn implies that repeated commutators produce a finite series in (26a), (26b) which can be computed explicitly after some tedious but straightforward calculations.

The second observation is that in (26a), (26b) when we replace $\mathcal{A}$ with $\mathcal{B}$ we obtain infinite series with a certain periodicity which allows for explicit summation. This can be expressed via a Lie algebra isomorphism. For symplectic matrices of the blocked form

$$
L:=\left(\begin{array}{cc}
d(x, y) & l(x, y) \\
k(x, y) & -d(y, x)
\end{array}\right)
$$

where $d, k$ and $l$ are kernels in $L^{2}$, and $k$ and $l$ are symmetric in $(x, y)$, we define the map from $L$ to quadratic polynomials is $\left(a, a^{*}\right)$ in the following manner,

$$
\mathcal{I}(L)=\frac{1}{2} \int d x d y\left\{\left(a_{x}, a_{x}^{*}\right)\left(\begin{array}{cc}
d(x, y) & l(x, y) \\
k(x, y) & -d(y, x)
\end{array}\right)\left(\begin{array}{c}
-a_{y}^{*} \\
a_{y}
\end{array}\right)\right\} .
$$

The crucial property of this map is the Lie algebra isomorphism

$$
\left[\mathcal{I}\left(L_{1}\right), \mathcal{I}\left(L_{2}\right)\right]=\mathcal{I}\left(\left[L_{1}, L_{2}\right]\right)
$$

thus any computation that involves commutations can be performed in the realm of symplectic matrices and then transfered to polynomials in $\left(a, a^{*}\right)$. In particular if we call $\mathcal{I}(H)=\mathcal{H}$ for a quadratic Hamiltonian and $\mathcal{I}(K)=\mathcal{B}$ then we have the two formulas below,

$$
\begin{aligned}
e^{\mathcal{B}} \mathcal{H} e^{-\mathcal{B}} & =\mathcal{I}\left(e^{K} H e^{-K}\right) \\
\left(\partial_{t} e^{\mathcal{B}}\right) e^{-\mathcal{B}} & =\mathcal{I}\left(\left(\partial_{t} e^{K}\right) e^{-K}\right) .
\end{aligned}
$$

Actually, to avoid the infinite trace in (29a), we write

$$
\begin{aligned}
e^{\mathcal{B}} \mathcal{H} e^{-\mathcal{B}} & =\mathcal{H}+\left[e^{\mathcal{B}}, \mathcal{H}\right] e^{-\mathcal{B}} \\
& =\mathcal{H}+\mathcal{I}\left(\left[e^{K}, H\right] e^{-K}\right)
\end{aligned}
$$


As a matter of fact if we define the following quardatic expressions,

$$
\begin{array}{lll}
\mathcal{D}_{x y}^{\dagger}:=a_{x} a_{y}^{*} & ; \quad \mathcal{D}_{x y}:=a_{x}^{*} a_{y} \\
\mathcal{Q}_{x y}^{*}:=a_{x}^{*} a_{y}^{*} & ; \quad Q_{x y}:=a_{x} a_{y}
\end{array}
$$

then we can write,

$\mathcal{I}(L)=-\frac{1}{2} \int d x d y\left\{d(x, y) \mathcal{D}_{x y}^{\dagger}+d(y, x) \mathcal{D}_{x y}+k(x, y) \mathcal{Q}_{x y}^{*}-l(x, y) \mathcal{Q}_{x y}\right\}$

Remark 2.7. Notice that $D_{x y}^{\dagger}=D_{y x}+\delta(x-y)$ thus we can write

$$
\begin{aligned}
\mathcal{I}(L)= & -\frac{1}{2} \int d x d y\left\{d(x, y) \mathcal{D}_{y x}+d(y, x) \mathcal{D}_{x y}+k(x, y) \mathcal{Q}_{x y}^{*}-l(x, y) \mathcal{Q}_{x y}\right\} \\
& -\frac{1}{2} \int d x\{d(x, x)\} .
\end{aligned}
$$

In our present formalism if we define the matrix

$$
K=\left(\begin{array}{ll}
0 & \bar{k} \\
k & 0
\end{array}\right),
$$

then we have that $\mathcal{I}(K)=\mathcal{B}$, see the expression in (12b). The exponential of $K$ can be computed,

$$
\begin{aligned}
e^{K} & =\left(\begin{array}{ll}
\operatorname{ch}(k) & \overline{\operatorname{sh}(k)} \\
\operatorname{sh}(k) & \overline{\operatorname{ch}(k)}
\end{array}\right) \quad \text { where, } \\
\operatorname{sh}(k) & :=k+\frac{1}{3 !} k \circ \bar{k} \circ k+\ldots, \\
\operatorname{ch}(k) & :=\delta(x-y)+\frac{1}{2 !} \bar{k} \circ k+\ldots,
\end{aligned}
$$

and $\circ$ indicates composition. For completeness and for the convenience of the reader we include in the appendix the derivation of (28), see also [20], [17].

Let us now proceed with the calculations. First look at the expression inside the parentheses in the reduced Hamiltonian (23). It is straightforward after repeated (but finite) commutations with $\mathcal{A}$ to come up with the expression below (see section 3 of [20]),

$$
\begin{aligned}
& \frac{1}{i}\left(\partial_{t} e^{\sqrt{N} \mathcal{A}}\right) e^{-\sqrt{N} \mathcal{A}}+e^{\sqrt{N} \mathcal{A}} \mathcal{H} e^{-\sqrt{N} \mathcal{A}} \\
& =N \mu_{0}+N^{1 / 2} \mathcal{P}_{1}+\mathcal{P}_{2}-N^{-1 / 2} \mathcal{P}_{3}-N^{-1} \mathcal{P}_{4}
\end{aligned}
$$

where $\mathcal{P}_{n}$ indicate polynomials of degree $n$ to be given explicitly below. The first term $\mu_{0}$ is a scalar which can be absorbed in the evolution as an extra phase factor. It is given by the commutators,

$$
\frac{1}{2 i}\left[\mathcal{A}, \partial_{t} \mathcal{A}\right]+\frac{1}{2}\left[\mathcal{A},\left[\mathcal{A}, \mathcal{H}_{1}\right]\right]-\frac{1}{4 !}[\mathcal{A},[\mathcal{A},[\mathcal{A},[\mathcal{A}, \mathcal{V}]]]]
$$


which reduce to the expression below,

$$
\begin{aligned}
\mu_{0} & :=\int d x\left\{\frac{1}{2 i}\left(\phi \bar{\phi}_{t}-\bar{\phi} \phi_{t}\right)-|\nabla \phi|^{2}\right\} \\
& -\frac{1}{2} \int d x d y\left\{v_{N}(x-y)|\phi(x)|^{2}|\phi(y)|^{2}\right\} .
\end{aligned}
$$

The first degree polynomial $\mathcal{P}_{1}$ arise from the commutators,

$$
\frac{1}{i} \partial_{t} \mathcal{A}+\left[\mathcal{A}, \mathcal{H}_{1}\right]-\frac{1}{3 !}[\mathcal{A},[\mathcal{A},[\mathcal{A}, \mathcal{V}]]]
$$

and it can be expressed as follows,

$$
\mathcal{P}_{1}=\int d x\left\{h(t, x) a_{x}^{*}+\bar{h}(t, x) a_{x}\right\}
$$

where $h:=-(1 / i) \partial_{t} \phi+\Delta \phi-\left(v_{N} *|\phi|^{2}\right) \phi$. The second degree polynomial consists of the terms

$$
\mathcal{H}_{1}-\frac{1}{2}[\mathcal{A},[\mathcal{A}, \mathcal{V}]]
$$

and can expressed

$$
\begin{aligned}
\mathcal{P}_{2} & =\frac{1}{2} \int d x d y\left\{-g_{N}(t, x, y) \mathcal{D}_{y x}-g_{N}(t, y, x) \mathcal{D}_{x, y}\right\} \\
& +\frac{1}{2} \int d x d y\left\{\bar{m}_{N}(t, x, y) \mathcal{Q}_{x y}+m_{N}(t, x, y) \mathcal{Q}_{x, y}^{*}\right\}
\end{aligned}
$$

where $g_{N}$ and $m_{N}$ are given by, see (15a), (15b)

$$
\begin{aligned}
g_{N}(t, x, y) & :=-\Delta_{x} \delta(x-y)+\left(v_{N} *|\phi|^{2}\right)(t, x) \delta(x-y) \\
& +v_{N}(x-y) \bar{\phi}(t, x) \phi(t, y) \\
m_{N}(x, y) & :=-v_{N}(x-y) \phi(x) \phi(y) .
\end{aligned}
$$

It is clear that $g_{N}$ and $m_{N}$ above depend on the number of particles $N$. Subsequently, for simplicity, we will suppres this subscript and recall it only when it is relevant in an argument. Let us define the two operators below

$$
\begin{aligned}
\mathcal{H}_{G} & :=\frac{1}{2} \int d x d y\left\{-g_{N}(t, x, y) \mathcal{D}_{y x}-g_{N}(t, y, x) \mathcal{D}_{x, y}\right\} \\
\mathcal{M} & :=\frac{1}{2} \int d x d y\left\{\bar{m}_{N}(t, x, y) \mathcal{Q}_{x y}+m_{N}(t, x, y) \mathcal{Q}_{x, y}^{*}\right\} \\
& =\mathcal{I}\left(\begin{array}{cc}
0 & \bar{m} \\
-m & 0
\end{array}\right)
\end{aligned}
$$

so that we can write $\mathcal{P}_{2}=\mathcal{H}_{G}+\mathcal{M}$. The relevance of this splitting will become clear shortly. The third and fourth degree polynomiasl 
arise from the commutators $[\mathcal{A}, \mathcal{V}]$ and $\mathcal{V}$ respectively and are given below

$$
\begin{aligned}
& \mathcal{P}_{3}:=\int d x d y\left\{v_{N}(x-y)\left(\phi(y) a_{x}^{*} a_{y}^{*} a_{x}+\bar{\phi}(y) a_{x}^{*} a_{x} a_{y}\right)\right\} \\
& \mathcal{P}_{4}:=(1 / 2) \int d x d y\left\{v_{N}(x-y) a_{x}^{*} a_{y}^{*} a_{x} a_{y}\right\} .
\end{aligned}
$$

The mean field approximation emerges from the first degree polynomial $\mathcal{P}_{1}$. Since $\mu_{0}$ can be absorbed into the evolution it is reasonable to pick the field $\phi$ so that $h(\phi)=0$. This leads to the evolution

$$
\frac{1}{i} \partial_{t} \phi-\Delta \phi+\left(v_{N} *|\phi|^{2}\right) \phi=0
$$

which is of Hartree type. The formal limit of the equation above is the cubic NLS where the constant in front of the nonlinear term is the integral of the potential $v$. If $\phi$ satisfies (36) then $\mu_{0}$ reduces to

$$
\mu_{0}=\frac{1}{2} \int d x d y\left\{v_{N}(x-y)|\phi(t, x)|^{2}|\phi(t, y)|^{2}\right\}
$$

Now we can compute the reduced Hamiltonian in (23) using the splitting in (34a), (34b). First let us first give a name to

$$
\mathcal{E}:=e^{\mathcal{B}}\left(\mathcal{P}_{3}+N^{-1 / 2} \mathcal{P}_{4}\right) e^{-B}
$$

which will be treated later as an error term. Now we can write, see (23)

$$
\begin{aligned}
\mathcal{H}_{\text {red }} & =\frac{1}{i}\left(\partial_{t} e^{\mathcal{B}}\right) e^{-\mathcal{B}}+\mathcal{H}_{G}+\left[e^{\mathcal{B}}, \mathcal{H}_{G}\right] e^{-\mathcal{B}}+e^{\mathcal{B}} \mathcal{I}(M) e^{-\mathcal{B}}+N \mu_{0} \\
& -e^{\mathcal{B}}\left(N^{-1 / 2} \mathcal{P}_{3}+N^{-1} \mathcal{P}_{4}\right) e^{-B}+N \mu_{0} \\
& =\mathcal{H}_{G}+\mathcal{I}\left((1 / i)\left(\partial_{t} e^{K}\right) e^{-K}+\left[e^{K}, G\right] e^{-K}+e^{K} M e^{-K}\right)-N^{-1 / 2} \mathcal{E}+N \mu_{0} \\
& =\mathcal{H}_{G}+\mathcal{I}(R)-N^{-1 / 2} \mathcal{E}+N \mu_{0}
\end{aligned}
$$

where $R$ is defined to be the expression,

$$
R:=(1 / i)\left(\partial_{t} e^{K}\right) e^{-K}+\left[e^{K}, G\right] e^{-K}+e^{K} M e^{-K} .
$$


For the convenience of the reader, let us recall our set up,

$$
\begin{aligned}
& K:=\left(\begin{array}{cc}
0 & \bar{k} \\
k & 0
\end{array}\right) \quad \text { and } e^{K}=\left(\begin{array}{cc}
\operatorname{ch}(k) & \overline{\operatorname{sh}(k)} \\
\operatorname{sh}(k) & \overline{\operatorname{ch}(k)}
\end{array}\right) \\
& \operatorname{sh}(k):=k+\frac{1}{3 !} k \circ \bar{k} \circ k+\ldots, \\
& \operatorname{ch}(k):=\delta(x-y)+\frac{1}{2 !} \bar{k} \circ k+\ldots, \\
& g(t, x, y):=-\Delta_{x} \delta(x-y)+\left(v_{N} *|\phi|^{2}\right)(t, x) \delta(x-y) \\
& \quad+v_{N}(x-y) \bar{\phi}(t, x) \phi(t, y), \\
& m(x, y):=-v_{N}(x-y) \phi(x) \phi(y) \quad \text { where } v_{N}(x)=N^{3 \beta} v\left(N^{\beta} x\right) \\
& G:=\left(\begin{array}{cc}
g & 0 \\
0 & -g^{T}
\end{array}\right) \quad \text { and } \quad M:=\left(\begin{array}{cc}
0 & \bar{m} \\
-m & 0
\end{array}\right) \\
& N_{u}:=\left(\begin{array}{cc}
I & 0 \\
0 & -I
\end{array}\right) \quad(\text { this corresponds to the Number operator }) \\
& \mathbf{S}(s):=\frac{1}{i} s_{t}+g^{T} \circ s+s \circ g \quad \text { and } \quad \mathbf{W}(p):=\frac{1}{i} p_{t}+\left[g^{T}, p\right] .
\end{aligned}
$$

Thus $\mathbf{S}$ describes a Shrödinger type evolution, while $\mathbf{W}$ is a Wigner type operator. These operators will emerge shortly. Recall the formula (39) that we derived earlier for the reduced Hamiltonian

$$
\mathcal{H}_{\text {red }}=\mathcal{H}_{G}+\mathcal{I}(R)-N^{-1 / 2} \mathcal{E}
$$

where $\mathcal{H}_{G}$ has only $a^{*} a$ terms (which annihilate the vacuum) and $R$ can be computed explicitly. In fact we have,

$$
\begin{aligned}
& R= \\
& \left(\frac{1}{i}\left(\begin{array}{cc}
\operatorname{ch}(k)_{t} & \overline{\operatorname{sh}(k)_{t}} \\
\operatorname{sh}(k)_{t} & \overline{\operatorname{ch}(k)_{t}}
\end{array}\right)\right. \\
& +\left(\begin{array}{cc}
{[\operatorname{ch}(k), g]-\overline{\operatorname{sh}} \circ m} & \left.-\overline{\operatorname{sh}(k)} \circ g^{T}-g \circ \overline{\operatorname{sh}(k)}+\operatorname{ch} \circ \bar{m}\right) \\
\operatorname{sh}(k) \circ g+g^{T} \circ \operatorname{sh}(k)-\overline{\operatorname{ch}} \circ m & -\left[\overline{\operatorname{ch}(k)}, g^{T}\right]+\operatorname{sh} \circ \bar{m}
\end{array}\right) \\
& \circ\left(\begin{array}{cc}
\operatorname{ch}(k) & -\overline{\operatorname{sh}(k)} \\
-\operatorname{sh}(k) & \overline{\operatorname{ch}(k)}
\end{array}\right)
\end{aligned}
$$

(matrix product, where kernel products mean compositions)

The condition that we would like to impose is that $R$ is block diagonal so that $\mathcal{I}(R)$ contains only terms of the form $a a^{*}$ and $a^{*} a$ so that, apart from a trace when we commute $a$ with $a^{*}$, we obtain an operator which annihilates the vacuum state. The remaining trace can be absorbed in 
the evolution as a phase factor. Thus our requirement is

$$
\frac{1}{i}\left(\frac{\partial}{\partial t} e^{K}\right) e^{-K}+\left[e^{K}, G\right] e^{-K}+e^{K} M e^{-K} \text { is block diagonal. }
$$

We proceed to show this equivalent to equations $(\underline{17 b}),(17 \mathrm{c})$. Let us make the elementary observations

$$
\begin{aligned}
& \left(\frac{\partial}{\partial t} e^{K}\right) e^{-K}=\frac{\partial}{\partial t} I-e^{K} \circ \frac{\partial}{\partial t} \circ e^{-K} \\
& {\left[e^{K}, G\right] e^{-K}=e^{K} G e^{-K}-G}
\end{aligned}
$$

so removing the part of (40) that is diagonal already we have the equivalent formulation of (40)

$$
e^{K}\left(-\frac{1}{i} \frac{\partial}{\partial t}+G+M\right) e^{-K} \text { is block diagonal. }
$$

Now we make the observation that a matrix is block-diagonal if and only if it commutes with the number operator matrix $N_{u}$, as well as (for arbitrary matrices $A$ and $B$ ) we have $\left[e^{K} A e^{-K}, B\right]=0$ if and only if $\left[A, e^{-K} B e^{K}\right]=0$, so our equation (41) reads,

$$
\left[\left(-\frac{1}{i} \frac{\partial}{\partial t}+G+M\right), e^{-K} N_{u} e^{K}\right]=0 .
$$

A direct calculation gives

$$
e^{-K} N_{u} e^{K}=\left(\begin{array}{cc}
\operatorname{ch}(2 k) & \overline{\operatorname{sh}(2 k)} \\
-\operatorname{sh}(2 k) & -\overline{\operatorname{ch}(2 k)}
\end{array}\right)
$$

after which is is straightforward to compute

$$
\left[-\frac{1}{i} \frac{\partial}{\partial t}+G, e^{-K} N_{u} e^{K}\right]=\left(\begin{array}{cc}
\overline{\mathbf{W}(\overline{\operatorname{ch}(2 k)})} & \overline{\mathbf{S}((\operatorname{sh}(2 k))} \\
\mathbf{S}(\overline{\operatorname{sh}(2 k))} & \mathbf{W}(\overline{\operatorname{ch}(2 k)})
\end{array}\right)
$$

and simlarly,

$$
\left[M, e^{-K} N_{u} e^{K}\right]=\left(\begin{array}{ll}
\overline{-m \circ \overline{\operatorname{sh}(2 k)}+\mathrm{sh}(2 k) \circ \bar{m}} & \overline{-m \circ \operatorname{ch}(2 k)-\overline{\operatorname{ch}(2 k)} \circ m} \\
-m \circ \operatorname{ch}(2 k)-\overline{\operatorname{ch}(2 k)} \circ m & -m \circ \overline{\operatorname{sh}(2 k)}+\operatorname{sh}(2 k) \circ \bar{m}
\end{array}\right)
$$

Finally combining the two formulas above we obtain, see (42), the linear pair of equations below

$$
\begin{aligned}
& \mathbf{S}(\operatorname{sh}(2 k))=m \circ \operatorname{ch}(2 k)+\overline{\operatorname{ch}(2 k)} \circ m \\
& \mathbf{W}(\overline{\operatorname{ch}(2 k)})=m \circ \overline{\operatorname{sh}(2 k)}-\operatorname{sh}(2 k) \circ \bar{m} .
\end{aligned}
$$

This completes the derivation of the evolution equations for the pair excitations and the mean field, namely (43a), (43b), together with (36) 
describe the evolution of $\phi$ and $k$ and are the equations in (17a), (17b) and (17c). In particular, we have proved that in that if $\phi, k$ satisfy these equations, then the energy estimate (24) holds.

\section{Estimates for the solution to the Hartree EQUATION}

This section adapts classical results for NLS due to Lin and Strauss [30], Ginibre and Velo [19], Bourgain [3], as well as Colliander, Keel, Staffilani, Takaoka and Tao [4] to the Hartree equation. Assume

$$
\begin{aligned}
& \frac{1}{i} \frac{\partial}{\partial t} \phi-\Delta \phi+\left(v_{N} *|\phi|^{2}\right) \phi=0 \\
& \phi(0, \cdot)=\phi_{0} .
\end{aligned}
$$

where $v \in C_{0}^{1}$ is non-negative, spherically symmetric, and decreasing. We recall the relevant conserved quantities, following the notation [21]:

$$
\begin{aligned}
\rho & :=(1 / 2)|\phi|^{2} ; \\
p_{j} & :=(1 / 2 i)\left(\phi \nabla_{j} \bar{\phi}-\bar{\phi} \nabla_{j} \phi\right) ; \quad p_{0}=(1 / 2 i)\left(\phi \partial_{t} \bar{\phi}-\bar{\phi} \partial_{t} \phi\right) ; \\
\sigma_{j k} & :=\nabla_{j} \bar{\phi} \nabla_{k} \phi+\nabla_{k} \bar{\phi} \nabla_{j} \phi ; \quad \sigma_{0 j}=\nabla_{j} \bar{\phi} \partial_{t} \phi+\partial_{t} \bar{\phi} \nabla_{j} \phi \\
\lambda & :=-\Im\left(\phi \partial_{t} \bar{\phi}\right)+|\nabla \phi|^{2}+\frac{1}{2}\left(v *|\phi|^{2}\right)|\phi|^{2} \\
= & \frac{1}{2}\left(\Delta|\phi|^{2}-\left(v *|\phi|^{2}\right)|\phi|^{2}\right) ; \\
e & :=|\nabla \phi|^{2}+\frac{1}{2}\left(v *|\phi|^{2}\right)|\phi|^{2} .
\end{aligned}
$$

The associated conservation laws are

$$
\begin{aligned}
& \partial_{t} \rho-\nabla_{j} p^{j}=0, \\
& \partial_{t} p_{j}-\nabla_{k}\left\{\sigma_{j}{ }^{k}-\delta_{j}{ }^{k} \lambda\right\}+l_{j}=0, \\
& \partial_{t} e-\nabla_{j} \sigma_{0}^{j}+l_{0}=0 .
\end{aligned}
$$

These laws express the conservation of mass, momentum and energy, respectively, where the vector $\left(l_{j}, l_{0}\right)$ is

$l_{j}:=2\left(\left(v_{N} * \rho\right) \rho_{j}-\left(v_{N} * \rho_{j}\right) \rho\right), \quad l_{0}:=2\left(\left(v_{N} * \rho\right) \rho_{0}-\left(v_{N} * \rho_{0}\right) \rho\right)$.

In the case of NLS, $v_{N}=\delta$ and $l_{j}, l_{0}$ are 0 .

We adapt the well-known method of interaction Morawetz estimates, due to Colliander, Keel, Staffilani, Takaoka and Tao, outlined in [4]. Start with

$$
Q(t)=\int\left(\nabla_{j} p^{j}(t, x) \rho(t, y)+\rho(t, x) \nabla_{j} p^{j}(t, x)\right)|x-y| d x d y .
$$


Using (45) we get

$$
\begin{aligned}
& \dot{Q}(t)=2 \int \nabla_{j} p^{j}(t, x) \nabla_{k} p^{k}(t, y)|x-y| d x d y \\
& +\int\left(\nabla_{j}\left(\nabla_{k}\left\{\sigma_{j}{ }^{k}(t, x)-\delta_{j}{ }^{k} \lambda(t, x)\right\}-l_{j}(t, x)\right) \rho(t, y)\right. \\
& \left.+\rho(t, x) \nabla_{j}\left(\left(\nabla_{k}\left\{\sigma_{j}{ }^{k}(t, y)-\delta_{j}{ }^{k} \lambda(t, y)\right\}-l_{j}(t, y)\right)\right)\right)|x-y| d x d y \\
& \geq \int(-\lambda(t, x) \rho(t, y)-\rho(t, x) \lambda(t, y)) \Delta|x-y| d x d y \text { (main term) } \\
& -\int\left(\left(\nabla_{j} l_{j}(t, x)\right) \rho(t, y)+\rho(t, x)\left(\nabla_{j} l_{j}(t, y)\right)\right)|x-y| d x d y \text { (error term.) }
\end{aligned}
$$

We have used the fact which we recall for the reader's convenience (see [4]), that

$$
\begin{aligned}
& \left(\nabla_{j} \nabla_{k} a\right)(x-y)\left(-2 p_{j}(t, x) p_{k}(t, y)+\sigma_{j}{ }^{k}(t, x) \rho(t, y)+\sigma_{j}{ }^{k}(t, y) \rho(t, x)\right) \\
& =\left(\nabla_{j} \nabla_{k} a\right)(x-y)\left(\left(\phi(x) \overline{\phi_{j}}(y)+\phi_{j}(x) \bar{\phi}(y)\right) \overline{\left(\phi(x) \overline{\phi_{j}}(y)+\phi_{j}(x) \bar{\phi}(y)\right)}\right. \\
& \left.+\left(\phi(x) \phi_{j}(y)-\phi_{j}(x) \phi(y)\right) \overline{\left(\phi(x) \phi_{j}(y)-\phi_{j}(x) \phi(y)\right)}\right) \geq 0
\end{aligned}
$$

where $a(x)=|x|$. It is easy to check

$$
\begin{aligned}
& \text { (main term) } \geq c\|\phi(t, \cdot)\|_{L^{4}}^{4} \\
& +2 \int\left(\left(v_{N} * \rho\right)(t, x) \rho(t, x) \rho(t, y)+\left(v_{N} * \rho\right)(t, y) \rho(t, y) \rho(t, x)\right) \Delta|x-y| d x d y \\
& \text { with } c>0
\end{aligned}
$$


We proceed to analyze the error term:

error term

$$
\begin{aligned}
& =-\int\left(\left(\nabla_{j} l_{j}(t, x)\right) \rho(t, y)+\rho(t, x)\left(\nabla_{j} l_{j}(t, y)\right)\right)|x-y| d x d y \\
& =-2 \int\left(\nabla_{j} l_{j}(t, x)\right) \rho(t, y)|x-y| d x d y \\
& =2 \int l_{j}(t, x) \rho(t, y) \frac{(x-y)^{j}}{|x-y|} d x d y \\
& =4 \int\left(\left(v_{N} * \rho\right)(t, x) \rho_{j}(t, x)-\left(v_{N} * \rho_{j}\right)(t, x) \rho(t, x)\right) \rho(t, y) \frac{(x-y)^{j}}{|x-y|} d x d y \\
& =4 \int v_{N}(x-z)\left(\left(\rho(t, z) \rho_{j}(t, x)-\rho_{j}(t, z) \rho(t, x)\right) \rho(t, y) \frac{(x-y)^{j}}{|x-y|} d x d y d z\right. \\
& =-8 \int v_{N}^{\prime}(|x-z|) \frac{(x-z)^{j}}{|x-z|}\left(\rho(t, z) \rho(t, x) \rho(t, y) \frac{(x-y)^{j}}{|x-y|} d x d y d z\right. \\
& -4 \int v_{N}(x-z) \rho(t, z) \rho(t, x) \rho(t, y) \partial_{x, j}\left(\frac{(x-y)^{j}}{|x-y|}\right) d x d y d z \\
& =-4 \int v_{N}^{\prime}(|x-z|)\left(\frac{(x-z)^{j}}{|x-z|} \frac{(x-y)^{j}}{|x-y|}+\frac{(z-x)^{j}}{|z-x|} \frac{(z-y)^{j}}{|z-y|}\right) \rho(t, z) \rho(t, x) \rho(t, y) \\
& -2 \int\left(\left(v_{N} * \rho\right)(t, x) \rho(t, x) \rho(t, y)+\left(v_{N} * \rho\right)(t, y) \rho(t, y) \rho(t, x)\right) \Delta|x-y| d x d y
\end{aligned}
$$

The next-to-last line is $\geq 0$ because of the assumption $v_{N}^{\prime} \leq 0$ and the elementary trigonometric inequality

$$
\begin{aligned}
& \frac{(x-z)^{j}}{|x-z|} \frac{(x-y)^{j}}{|x-y|}+\frac{(z-x)^{j}}{|z-x|} \frac{(z-y)^{j}}{|z-y|} \\
& =\cos \left(\theta_{1}\right)+\cos \left(\theta_{2}\right) \geq 0
\end{aligned}
$$

The last line is negative, but cancels part of the main term. Thus

$$
(\text { main term })+(\text { error term }) \geq c\|\phi\|_{L^{4}}^{4}
$$

Since $Q(t)$ is bounded uniformly in time by $\left\|\phi_{0}\right\|_{H^{1}}^{4}$, we have shown the following proposition.

Proposition 3.1. Let $\phi$ be a solution to the Hartree equation (44). There exists $C$ depending only on $\left\|\phi_{0}\right\|_{H^{1}}$ such that

$$
\|\phi\|_{L^{4}\left([0, \infty) \times \mathbb{R}^{3}\right)} \leq C
$$

and, as an immediate consequence of conservation of energy,

$$
\|\phi\|_{L^{8}[0, \infty) L^{4}\left(\mathbb{R}^{3}\right)} \leq C .
$$


Remark 3.2. It was shown by Bourgain in [3] that if $\phi$ is a solution to cubic NLS, then there exists $C_{s}$ depending only on $\left\|\phi_{0}\right\|_{H^{s}}$ such that

$$
\|\phi(t, \cdot)\|_{H^{s}} \leq C_{s} \forall t
$$

Using the above Morawetz estimate (which was not yet discovered when Bourgain did this work), we can easily prove the same for our Hartree equation.

Proposition 3.3. Let $\phi$ be a solution to the equation (44). There exists $C_{s}$ depending only on $\left\|\phi_{0}\right\|_{H^{s}}$ such that such that

$$
\|\phi(t, \cdot)\|_{H^{s}} \leq C_{s}
$$

uniformly in time.

Proof. Split $[0, \infty)$ into finitely many intervals $I_{k}$ where

$$
\|\phi\|_{L^{8}\left(I_{k}\right) L^{4}\left(\mathbb{R}^{3}\right)} \leq \epsilon
$$

where $\epsilon$ is to be prescribed later. Differentiating (44)

$$
\begin{aligned}
& \frac{1}{i} \frac{\partial}{\partial t} D^{s} \phi-\Delta D^{s} \phi=-D_{s}\left(\left(v_{N} *|\phi|^{2}\right) \phi\right) \\
& \text { where } \\
& D_{s}\left(\left(v_{N} *|\phi|^{2}\right) \phi\right)=\left(v_{N} *|\phi|^{2}\right) D^{s} \phi+\text { similar and easier terms. }
\end{aligned}
$$

For the first interval, $I_{1}$, we get, using the $L^{8 / 3} L^{4}$ Strichartz estimate,

$$
\begin{aligned}
& \left\|D^{s} \phi\right\|_{L^{8 / 3}\left(I_{1}\right) L^{4}\left(\mathbb{R}^{3}\right)} \leq C\left\|\phi_{0}\right\|_{H^{s}}+C\left\|\left(v_{N} *|\phi|^{2}\right) D^{s} \phi\right\|_{L^{8 / 5\left(I_{1}\right)} L^{4 / 3}\left(\mathbb{R}^{3}\right)} \\
& \leq C_{1}\left\|\phi_{0}\right\|_{H^{s}}+C_{2}\|\phi\|_{L^{8}\left(I_{1}\right) L^{4}\left(\mathbb{R}^{3}\right)}^{2}\left\|D^{s} \phi\right\|_{L^{8 / 3}\left(I_{1}\right) L^{4}\left(\mathbb{R}^{3}\right)} \cdot
\end{aligned}
$$

At this stage, we pick $\epsilon$ so that $C_{2} \epsilon^{2} \leq \frac{1}{2}$ to conclude

$$
\left\|D^{s} \phi\right\|_{L^{8 / 3\left(I_{1}\right) L^{4}\left(\mathbb{R}^{3}\right)}} \leq 2 C_{1}\left\|\phi_{0}\right\|_{H^{s}} .
$$

In turn, this allows us to control the inhomogeneity of (47)

$$
\begin{aligned}
& \left\|\left(v_{N} *|\phi|^{2}\right) D^{s} \phi\right\|_{L^{8 / 5}\left(I_{1}\right) L^{4 / 3}\left(\mathbb{R}^{3}\right)} \\
& \leq\|\phi\|_{L^{8}\left(I_{1}\right) L^{4}\left(\mathbb{R}^{3}\right)}^{2}\left\|D^{s} \phi\right\|_{L^{8 / 3}\left(I_{1}\right) L^{4}\left(\mathbb{R}^{3}\right)} \\
& \leq C\left\|\phi_{0}\right\|_{H^{s}}^{3}
\end{aligned}
$$

and therefore

$$
\begin{aligned}
& \|\phi(t, \cdot)\|_{H^{s}} \leq\left\|\phi_{0}\right\|_{H^{s}}+C\|\phi\|_{L^{8}\left(I_{1}\right) L^{4}\left(\mathbb{R}^{3}\right)}^{2}\left\|D^{s} \phi\right\|_{L^{8 / 3}\left(I_{1}\right) L^{4}\left(\mathbb{R}^{3}\right)} \\
& \leq C\left\|\phi_{0}\right\|_{H^{s}}^{3}
\end{aligned}
$$

for all $t \in I_{1}$. Repeating the process finitely many times, we are done. 
If we assume the data $\phi_{0}$ and sufficiently many derivatives are not only in $L^{2}$ but also in $L^{1}$, we can also get decay.

Corollary 3.4. Let $\phi$ be a solution to (44). There exists $C$ depending only on $\left\|\phi_{0}\right\|_{W^{k, 1}}$ for $k$ sufficiently large such that

$$
\begin{aligned}
& \|\phi(t, \cdot)\|_{L^{\infty}} \leq \frac{C}{t^{\frac{3}{2}}} \\
& \text { and also } \\
& \left\|\partial_{t} \phi(t, \cdot)\right\|_{L^{\infty}} \leq \frac{C}{t^{\frac{3}{2}}}
\end{aligned}
$$

Proof. The proof follows the outline of [30], except that we have two modern ingredients which were not available to Lin and Strauss in 1977:

$$
\begin{aligned}
& \|\phi\|_{C^{s}\left(\mathbb{R}^{3+1}\right)} \leq C_{s} s \in \mathbb{N} \\
& \|\phi\|_{L^{4}\left(\mathbb{R}^{3+1}\right)} \leq C
\end{aligned}
$$

This implies that $\|\phi(t, \cdot)\|_{L^{\infty}\left(\mathbb{R}^{3}\right)} \rightarrow 0$ as $t \rightarrow \infty$. Indeed,

$$
\begin{aligned}
& \left\|\nabla\left(\phi^{2}\right)\right\|_{L^{4}\left([n, n+1] \times \mathbb{R}^{3}\right)} \\
& \leq 2\|\nabla \phi\|_{L^{\infty}\left(\mathbb{R}^{3+1}\right)}\|\phi\|_{L^{4}\left([n, n+1] \times \mathbb{R}^{3}\right)} \rightarrow 0
\end{aligned}
$$

This implies $\|\phi\|_{L^{p}\left([n, n+1] \times \mathbb{R}^{3}\right)} \rightarrow 0$ for any fixed $4<p<\infty$. Repeating the process one more time implies $\|\phi(t, \cdot)\|_{L^{\infty}\left(\mathbb{R}^{3}\right)} \rightarrow 0$.

We solve (44) by Duhamel's formula and use the standard $L^{\infty} L^{1}$ decay estimate for the linear equation. We use the following estimate:

$$
\begin{aligned}
& \left\|e^{i(t-s) \Delta}\left(\left(v *|\phi|^{2}\right) \phi(s)\right)\right\|_{L^{\infty}} \\
& \leq \frac{C}{|t-s|^{3 / 2}}\left\|\left(v *|\phi|^{2}\right) \phi(s)\right\|_{L^{1}} \leq \frac{C}{|t-s|^{3 / 2}}\|\phi(s, \cdot)\|_{L^{\infty}}
\end{aligned}
$$

We would also like to estimate $\left\|e^{i(t-s) \Delta}\left(\left(v *|\phi|^{2}\right) \phi(s)\right)\right\|_{L^{\infty}}$ by $\left\|\nabla e^{i(t-s) \Delta}\left(\left(v *|\phi|^{2}\right) \phi(s)\right)\right\|_{L^{3}}$. This is a false end-point, but becomes true if one replaces 3 by $3+\epsilon$. To keep numbers easy, skip the $\epsilon$ and notice first that

$$
\begin{aligned}
& \left\|\nabla e^{i(t-s) \Delta}\left(\left(v *|\phi|^{2}\right) \phi(s)\right)\right\|_{L^{3}} \leq \frac{C}{|t-s|^{1 / 2}}\left\|\nabla\left(v *|\phi|^{2}\right) \phi(s)\right\|_{L^{3 / 2}} \\
& \leq \frac{C}{|t-s|^{1 / 2}}\|\nabla \phi\|_{L^{2}}\left\|\phi^{2}\right\|_{L^{6}} \leq \frac{C}{|t-s|^{1 / 2}}\|\phi(s, \cdot)\|_{L^{4}}^{2 / 3}\|\phi(s, \cdot)\|_{L^{\infty}}^{4 / 3} \\
& \leq \frac{C}{|t-s|^{1 / 2}}\|\phi(s, \cdot)\|_{L^{\infty}}^{4 / 3}
\end{aligned}
$$


Now, using $3+\epsilon$ rather than 3 leads to an estimate of the form

$$
\left\|e^{i(t-s) \Delta}\left(\left(v *|\phi|^{2}\right) \phi(s)\right)\right\|_{L^{\infty}} \leq \frac{C}{|t-s|^{1 / 2+\epsilon^{\prime}}}\|\phi(s, \cdot)\|_{L^{\infty}}^{4 / 3-\epsilon^{\prime \prime}}
$$

Combining (49) and (51) we get: There exists a kernel $\in L^{1}([0, \infty))$ and $\delta>0$ such that

$$
\left\|e^{i(t-s) \Delta}\left(\left(v *|\phi|^{2}\right) \phi(s)\right)\right\|_{L^{\infty}} \leq k(t-s)\|\phi(s, \cdot)\|_{L^{\infty}}^{1+\delta}
$$

Putting all together

$$
\|\phi(t, \cdot)\|_{L^{\infty}} \leq \frac{C}{t^{3 / 2}}\left\|\phi_{0}\right\|_{L^{1}}+\int_{0}^{t / 2} \frac{C}{|t-s|^{3 / 2}}\|\phi(s, \cdot)\|_{L^{\infty}} d s+\int_{t / 2}^{t} k(t-s)\|\phi(s, \cdot)\|_{L^{\infty}}^{1+\delta} d s
$$

Denoting $M(t)=\sup _{0<s<t}\left(1+s^{3 / 2}\right)\|\phi(s, \cdot)\|_{L^{\infty}}$, We have, for $t>1$,

$M(t) \leq C\left\|\phi_{0}\right\|_{L^{1}}+\frac{C}{\left(1+|t|^{3 / 2}\right)} \int_{0}^{t / 2} \frac{1}{\left(1+|s|^{3 / 2}\right)} M(s) d s+C \sup _{t / 2<s<t}\|u(s, \cdot)\|_{L^{\infty}}^{\delta} M(t)$

The last term can be absorbed in $M(t)$, and the result follows by Gronwall's inequality. Now that we know that $\|\phi(s, \cdot)\|_{L^{\infty}} \leq \frac{C}{1+s^{3 / 2}}$, it is very easy to estimate $\partial_{t} \phi$. We use (49) and (52) (with $3+\epsilon$ replacing 3 ), as well as the fact that all norms $\left\|\partial^{\alpha} \phi(s, \cdot)\right\|_{L^{p}} \leq C_{\alpha, p}$ uniformly in $s$, for all $\mathrm{p}(k) \geq 2$. This is a consequence of Proposition (3.3) (boundedness of the $H^{s}$ norms).

$$
\begin{aligned}
& \left\|\partial_{t} \phi(t, \cdot)\right\|_{L^{\infty}} \leq \frac{C}{t^{3 / 2}}\left\|\partial_{t} \phi_{0}\right\|_{L^{1}}+\int_{0}^{t}\left\|e^{i(t-s) \Delta} \partial_{s}\left(\left(v *|\phi|^{2}\right) \phi(s)\right)\right\|_{L^{\infty}} \\
& \leq \frac{C}{t^{3 / 2}}+C \int_{0}^{t-1} \frac{1}{1+|t-s|^{3 / 2}}\left\|\partial_{s}\left(\left(v *|\phi|^{2}\right) \phi(s)\right)\right\|_{L^{1}} d s \\
& +C \int_{t-1}^{t} \frac{1}{1+|t-s|^{1 / 2+\epsilon}}\left\|\nabla \partial_{s}\left(\left(v *|\phi|^{2}\right) \phi(s)\right)\right\|_{L^{3 / 2-\epsilon^{\prime}}} d s \\
& \leq \frac{C}{t^{3 / 2}}+C \int_{0}^{t-1} \frac{1}{1+|t-s|^{3 / 2}}\left\|\partial_{s}\left(\left(v *|\phi|^{2}\right) \phi(s)\right)\right\|_{L^{1}} d s \\
& +C \int_{t-1}^{t} \frac{1}{1+|t-s|^{1 / 2+\epsilon} \| \nabla \partial_{s}}\left(\left(v *|\phi|^{2}\right) \phi(s)\right) \|_{L^{3 / 2-\epsilon^{\prime}}} d s \\
& \leq \frac{C}{t^{3 / 2}}+C \int_{0}^{t-1} \frac{1}{1+|t-s|^{3 / 2}}\|\phi(s)\|_{L^{\infty}} d s \\
& +C \int_{t-1}^{t} \frac{1}{1+|t-s|^{1 / 2+\epsilon}}\|\phi(s)\|_{L^{\infty}} d s
\end{aligned}
$$

If we estimate $\|\phi(s)\|_{L^{\infty}}$ using (48a), we are done. 
By interpolating with the $L^{2}$ uniform bound we get the next Corollary.

Corollary 3.5. Let $\phi$ be a solution to (44). There exists $C$ depending only on $\left\|\phi_{0}\right\|_{W^{k, 1}}$ for $k$ sufficiently large such that

$$
\|\phi(t, \cdot)\|_{L^{3}}+\left\|\partial_{t} \phi(t, \cdot)\right\|_{L^{3}} \leq \frac{C}{1+t^{\frac{1}{2}}} .
$$

\section{Estimates FOR THE PAIR EXCITATIONS}

Define $\operatorname{ch}(2 k):=\delta+p_{2}, \operatorname{sh}(2 k):=s_{2}$, and also $\operatorname{ch}(k):=\delta+p_{1}$, $\operatorname{sh}(2 k):=s_{1}$ so that, see (43a), (43b) become

$$
\begin{aligned}
& \mathbf{S}\left(s_{2}\right)=2 m+m \circ p_{2}+\bar{p}_{2} \circ m \\
& \mathbf{W}\left(\bar{p}_{2}\right)=m \circ \bar{s}_{2}-s_{2} \circ \bar{m} \\
& s_{2}(0, \cdot)=p_{2}(0, \cdot)=0
\end{aligned}
$$

The goal of this section is to prove the following theorem.

Theorem 4.1. Assume $\phi_{0} \in W^{k, 1}$ for $k$ sufficiently large. The following estimates hold:

$$
\left\|s_{2}(t, \cdot)\right\|_{L^{2}\left(\mathbb{R}^{6}\right)}+\left\|p_{2}(t, \cdot)\right\|_{L^{2}\left(\mathbb{R}^{6}\right)} \leq C \log (1+t)
$$

where $C$ depends on $\|\phi(0, \cdot)\|_{W^{k, 1}}$ for some finite $k$. A similar result holds for the higher time derivatives, but we will not use it or prove it.

An immediate corollary is of the above theorem is,

Corollary 4.2. The following estimates hold:

$$
\begin{aligned}
\left\|s_{1}(t, \cdot)\right\|_{L^{2}\left(\mathbb{R}^{6}\right)} & \leq C \log (1+t) \\
\left\|p_{1}(t, \cdot)\right\|_{L^{2}\left(\mathbb{R}^{6}\right)} & \leq C \log (1+t) \\
\int\left|p_{1}(x, x)\right| d x & \leq C \log ^{2}(1+t) .
\end{aligned}
$$

Proof. (of corollary (4.2)) Since $\operatorname{sh}(2 k)=2 \operatorname{sh}(k) \circ \operatorname{ch}(k)$, we get

$$
\begin{aligned}
& \left\|s_{1}(t, \cdot)\right\|_{L^{2}\left(\mathbb{R}^{6}\right)} \leq \frac{1}{2}\left\|s_{2}(t, \cdot)\right\|_{L^{2}\left(\mathbb{R}^{6}\right)}\left\|\operatorname{ch}(k)^{-1}\right\|_{\text {operator }} \\
& \leq \frac{1}{2}\left\|s_{2}(t, \cdot)\right\|_{L^{2}\left(\mathbb{R}^{6}\right)} \cdot
\end{aligned}
$$


We also have $p_{1}(x, x) \geq 0, p_{1} \circ p_{1}(x, x) \geq 0$, so taking traces in the relation

$$
p_{1} \circ p_{1}+2 p_{1}=\bar{s}_{1} \circ s_{1}
$$

gives the other estimates.

Before starting the proof of Theorem (4.1) , we need some preliminary lemmas.

Lemma 4.3. Recall $m(t, x, y)=-v_{N}(x-y) \phi(t, x) \phi(t, y)$. Then there exists $C$ such that

$$
\begin{aligned}
& \int \frac{\left|\widehat{m_{N}}(t, \xi, \eta)\right|^{2}}{\left(|\xi|^{2}+|\eta|^{2}\right)^{2}} d \xi d \eta \leq C\|\phi(t, \cdot)\|_{L^{3}}^{4} \\
& \text { and also } \\
& \int \frac{\left|\partial_{t} \widehat{m_{N}}(t, \xi, \eta)\right|^{2}}{\left(|\xi|^{2}+|\eta|^{2}\right)^{2}} d \xi d \eta \leq\|\phi(t, \cdot)\|_{L^{3}}^{2}\left\|\partial_{t} \phi(t, \cdot)\right\|_{L^{3}}^{2} .
\end{aligned}
$$

Similar estimates hold for higher time derivatives.

Proof. Write

$$
v_{N}(x-y) \phi(t, x) \phi(t, y)=\int \delta(x-y-z) v_{N}(z) \phi(t, x) \phi(t, y) d z .
$$

The Fourier transform of $\delta(x-y-z) \phi(t, x) \phi(t, y)$ is easily computed to be

$$
e^{i z \cdot \eta} \widehat{\phi \phi_{z}}(t, \xi+\eta)
$$

where we denote $\phi_{z}(x)=\phi(x-z)$. Thus

$$
\begin{aligned}
& \left|\widehat{m_{N}}(t, \xi, \eta)\right|^{2}=\left|\int v_{N}(z) e^{i z \cdot \eta} \widehat{\phi \phi_{z}}(t, \xi+\eta) d z\right|^{2} \\
& \leq\left\|v_{N}\right\|_{L^{1}} \int\left|v_{N}(z) \| \widehat{\phi \phi_{z}}(t, \xi+\eta)\right|^{2} d z
\end{aligned}
$$

Thus, after a change of variables, the left hand side of (55a) is dominated by

$$
\begin{aligned}
& \int\left|v_{N}(z)\right| \frac{\left|\widehat{\phi \phi_{z}}(t, \xi)\right|^{2}}{\left(|\xi|^{2}+|\eta|^{2}\right)^{2}} d \xi d \eta d z \\
& \leq C \int\left|v_{N}(z)\right| \frac{\left|\widehat{\phi \phi_{z}}(t, \xi)\right|^{2}}{|\xi|} d \xi d z \\
& \leq C \int\left|v_{N}(z)\right|\|\phi\|_{L^{3}}^{4} d z=C\|\phi\|_{L^{3}}^{4} .
\end{aligned}
$$


We have used the fact that

$$
\begin{aligned}
& \int \frac{\left|\widehat{\phi \phi_{z}}(t, \xi)\right|^{2}}{|\xi|} d \xi \leq C\left\|D_{x}^{-1 / 2}\left(\phi \phi_{z}\right)\right\|_{L^{2}}^{2} \\
& \leq C\left\|\phi \phi_{z}\right\|_{L^{3 / 2}}^{2} \text { (by Hardy-Littlewood-Sobolev) } \\
& \leq C\|\phi\|_{L^{3}}^{4} .
\end{aligned}
$$

The proof of (55b) is similar.

Lemma 4.4. Let $s_{a}^{0}$ be the solution to

$$
\begin{aligned}
& \left(\frac{1}{i} \frac{\partial}{\partial t}-\Delta_{\mathbb{R}^{6}}\right) s_{a}^{0}(t, x, y)=2 m(t, x, y) \\
& s_{a}^{0}(0, x, y)=0 .
\end{aligned}
$$

Then

$$
\begin{aligned}
& \qquad s_{a}^{0}(t, \cdot) \|_{L^{2}\left(\mathbb{R}^{6}\right)} \\
& \quad \leq C\left(\|\phi(0, \cdot)\|_{L^{3}}^{2}+\|\phi(t, \cdot)\|_{L^{3}}^{2}+\int_{0}^{t}\|\phi(s, \cdot)\|_{L^{3}}\left\|\partial_{s} \phi(s, \cdot)\right\|_{L^{3}} d s\right) \\
& \quad \leq C \log (1+t) \\
& \text { where } C \text { depends only on }\left\|\phi_{0}\right\|_{W^{k, 1}} \text {. }
\end{aligned}
$$

Proof. Solving (56) by Duhamel's formula we get

$$
\begin{aligned}
& \left\|s_{a}^{0}(t, \cdot)\right\|_{L^{2}\left(\mathbb{R}^{6}\right)} \\
& \leq C\left\|\int_{0}^{t} e^{i s\left(|\xi|^{2}+|\eta|^{2}\right)} \hat{m}(s, \xi, \eta) d s\right\|_{L^{2}\left(\mathbb{R}^{6}\right)} \\
& \leq C\left(\left\|\frac{\hat{m}(0, \xi, \eta)}{|\xi|^{2}+|\eta|^{2}}\right\|_{L^{2}\left(\mathbb{R}^{6}\right)}+\left\|\frac{\hat{m}(t, \xi, \eta)}{|\xi|^{2}+|\eta|^{2}}\right\|_{L^{2}\left(\mathbb{R}^{6}\right)}\right) \\
& +\left\|\int_{0}^{t} e^{i s\left(|\xi|^{2}+|\eta|^{2}\right)} \frac{\frac{\partial}{\partial s} \hat{m}(s, \xi, \eta)}{|\xi|^{2}+|\eta|^{2}} d s\right\|_{L^{2}\left(\mathbb{R}^{6}\right)} .
\end{aligned}
$$

The terms (57) are estimated directly by Lemma (4.3). For the last term (58) move the norm inside the integral and use Lemma (4.3) as well as Corollary (3.5).

Lemma 4.5. Let $s_{a}$ be the solution to

$$
\begin{aligned}
& \mathbf{S}\left(s_{a}\right)=2 m(t, x, y) \\
& s_{a}(0, x, y)=0 .
\end{aligned}
$$

Then

$$
\left\|s_{a}(t, \cdot)\right\|_{L^{2}\left(\mathbb{R}^{6}\right)} \leq C \log (1+t)
$$

where $C$ depends on $\left\|\phi_{0}\right\|_{W k, 1}$. 
Proof. Let $V$ be the "potential" part of $\mathbf{S}$, so that

$$
\mathbf{S}=\frac{1}{i} \frac{\partial}{\partial t}-\Delta_{\mathbb{R}^{6}}+V
$$

The operator $V$ is bounded from $L^{2}$ to $L^{2}$, with norm bounded by $\|\phi(t, \cdot)\|_{L^{\infty}}^{2} \leq \frac{C}{1+t^{3}}$ (by Corollary (3.4) $)$. Explicitly,

$$
\begin{aligned}
& V(u)(t, x, y)=\left(\left(v_{N} *|\phi|^{2}\right)(t, x)+\left(v_{N} *|\phi|^{2}\right)(t, y)\right) u(t, x, y) \\
& +\int v_{N}(x-z) \bar{\phi}(t, x) \phi(t, z) u(z, y) d z+\int u(x, z) v_{N}(z-y) \phi(t, z) \bar{\phi}(t, y) d z .
\end{aligned}
$$

Write

$$
s_{a}=s_{a}^{0}+s_{a}^{1}
$$

where $s_{a}^{0}$ is as in the previous lemma,

$$
\frac{1}{i} \frac{\partial}{\partial t} s_{a}^{0}(t, x, y)-\Delta_{\mathbb{R}^{6}} s_{a}^{0}(t, x, y)=2 m .
$$

Then $s_{a}^{1}$ satisfies the equation

$$
\mathbf{S}\left(s_{a}^{1}\right)=-V\left(s_{a}^{0}(t, \cdot)\right) .
$$

Both $s_{a}^{0}$ and $s_{a}^{1}$ are zero initially, and the estimate is already known for $s_{a}^{0}$. . We apply energy estimates to (60). Recall

$\mathbf{W}\left(s_{a}^{1} \circ \overline{s_{a}^{1}}\right)=\mathbf{S}\left(s_{a}^{1}\right) \circ \overline{s_{a}^{1}}-s_{a}^{1} \circ \overline{\mathbf{S}\left(s_{a}^{1}\right)}=-V\left(s_{a}^{0}(t, \cdot)\right) \circ \overline{s_{a}^{1}}+s_{a}^{1} \circ \overline{V\left(s_{a}^{0}(t, \cdot)\right)}$.

Taking traces, we get

$$
\begin{aligned}
& \frac{\partial}{\partial t}\left\|s_{a}^{1}\right\|_{L^{2}\left(\mathbb{R}^{6}\right)}^{2} \leq 2\left\|V\left(s_{a}^{0}(t, \cdot)\right)\right\|_{L^{2}\left(\mathbb{R}^{6}\right)}\left\|s_{a}^{1}\right\|_{L^{2}\left(\mathbb{R}^{6}\right)} \\
& \leq \frac{C}{1+t^{3}}\left\|s_{a}^{0}(t, \cdot)\right\|_{L^{2}\left(\mathbb{R}^{6}\right)}\left\|s_{a}^{1}\right\|_{L^{2}\left(\mathbb{R}^{6}\right)} \\
& \leq \frac{C \log (1+t)}{1+t^{3}}\left\|s_{a}^{1}(t, \cdot)\right\|_{L^{2}\left(\mathbb{R}^{6}\right)} .
\end{aligned}
$$

Integrating, we get the estimate

$$
\left\|s_{a}^{1}(t, \cdot)\right\|_{L^{2}\left(\mathbb{R}^{6}\right)} \leq C
$$

which implies the claim.

We are ready for the proof of Theorem (4.1).

Proof. Write $s_{2}=s_{a}+s_{e}$ where $\mathbf{S}\left(s_{a}\right)=2 m$, as in the previous lemma. The kernels $s_{e}$ and $p_{2}$ satisfy the following less singular system:

$$
\begin{aligned}
& \mathbf{S}\left(s_{e}\right)=m \circ p_{2}+\bar{p}_{2} \circ m \\
& \mathbf{W}\left(\bar{p}_{2}\right)=\left(m \circ \overline{s_{a}}-s_{a} \circ \bar{m}\right)+m \circ \overline{s_{e}}-s_{e} \circ \bar{m} .
\end{aligned}
$$


Using lemma (4.5) to estimate $\left\|s_{a}(t, \cdot)\right\|_{L^{2}}$ and defining

$$
M(t, \cdot)=m \circ \overline{s_{a}}-s_{a} \circ \bar{m}
$$

we have

$$
\begin{aligned}
& \mathbf{S}\left(s_{e}\right)=m \circ p_{2}+\bar{p}_{2} \circ m \\
& \mathbf{W}\left(\bar{p}_{2}\right)=M+m \circ \overline{s_{e}}-s_{e} \circ \bar{m}
\end{aligned}
$$

where

$$
\|M(t, \cdot)\|_{L^{2}\left(\mathbb{R}^{6}\right)} \leq \frac{C \log (1+t)}{1+t^{3}}
$$

since

$$
\|m \circ s\|_{L^{2}} \leq C\|\phi\|_{L^{\infty}}^{2}\|s\|_{L^{2}} \leq \frac{C}{1+t^{3}}\|s\|_{L^{2}} .
$$

Using the general formulas

$$
\begin{aligned}
\mathbf{W}(s \circ \bar{s}) & =\mathbf{S}(s) \circ \bar{s}-s \circ \overline{\mathbf{S}(s)} \\
\mathbf{W}(p \circ p) & =\mathbf{W}(p) \circ p+p \circ \mathbf{W}(p)
\end{aligned}
$$

and taking traces we get

$$
\begin{aligned}
& \frac{\partial}{\partial t}\left(\left\|s_{e}(t, \cdot)\right\|_{L^{2}\left(\mathbb{R}^{6}\right)}^{2}+\left\|p_{2}(t, \cdot)\right\|_{L^{2}\left(\mathbb{R}^{6}\right)}^{2}\right) \\
& \leq \frac{C}{1+t^{3}}\left(\left\|p_{2}(t, \cdot)\right\|_{L^{2}\left(\mathbb{R}^{6}\right)}\left\|s_{e}(t, \cdot)\right\|_{L^{2}\left(\mathbb{R}^{6}\right)}\right)+C\|M(t, \cdot)\|_{L^{2}\left(\mathbb{R}^{6}\right)}\left\|p_{2}(t, \cdot)\right\|_{L^{2}\left(\mathbb{R}^{6}\right)}
\end{aligned}
$$

which leads to the desired estimate. Explicitly, define

$$
E^{2}(t)=\left\|s_{e}(t, \cdot)\right\|_{L^{2}\left(\mathbb{R}^{6}\right)}^{2}+\left\|p_{2}(t, \cdot)\right\|_{L^{2}\left(\mathbb{R}^{6}\right)}^{2}
$$

then

$$
\frac{\partial}{\partial t} E(t) \leq C\left(\frac{1}{1+t^{3}} E(t)+\|M(t, \cdot)\|_{L^{2}}\right)
$$

thus $E(t)$ is uniformly bounded. Also, $\left\|p_{2}\right\|_{L^{2}}$ stays uniformly bounded and the logarithmic growth of $\left\|s_{2}\right\|_{L^{2}}$ can be traced back to $\left\|s_{a}^{0}\right\|_{L^{2}}$ from Lemma (4.4).

\section{List OF ALL THE ERROR TERMS}

Our purpose in the present section is to compute explicitly all the error terms and show how they can be estimated. Fortunately there are only a few terms for which one should be carefull, the rest being easier to handle.

Let us recall here briefly our basic strategy, which is to define

$$
\left|\psi_{\text {red }}\right\rangle:=e^{\mathcal{B}(k(t))} e^{\sqrt{N} \mathcal{A}(\phi(t))} e^{i t \mathcal{H}} e^{-\sqrt{N} \mathcal{A}(\phi(0))}|0\rangle
$$


and compute $\mathcal{H}_{\text {red }}$ such that $(1 / i) \partial_{t}\left|\psi_{\text {red }}\right\rangle=\mathcal{H}_{\text {red }}\left|\psi_{\text {red }}\right\rangle$.

$$
\begin{aligned}
& \mathcal{H}_{\text {red }}=\frac{1}{i} \frac{\partial}{\partial t}\left(e^{\mathcal{B}(k(t))}\right) e^{-\mathcal{B}(k(t))} \\
& +e^{\mathcal{B}(k(t))}\left(\left(\frac{1}{i} \frac{\partial}{\partial t} e^{\sqrt{N} \mathcal{A}(\phi(t))}\right) e^{-\sqrt{N} \mathcal{A}(\phi(t))}+e^{\sqrt{N} \mathcal{A}(\phi(t))} H e^{-\sqrt{N} \mathcal{A}(\phi(t))}\right) e^{-\mathcal{B}(k(t))} \\
& =N \mu_{0}(t) \\
& +N^{1 / 2} e^{\mathcal{B}(k(t))} \int d x\left\{h(\phi(t, x)) a_{x}^{*}+\bar{h}(\phi(t, x))\right\} e^{-\mathcal{B}(k(t))} \\
& +\frac{1}{i} \frac{\partial}{\partial t}\left(e^{\mathcal{B}(k(t))}\right) e^{-\mathcal{B}(k(t)}+e^{\mathcal{B}(k(t))}\left(\mathcal{H}_{1}-\frac{1}{2}[[\mathcal{A},[\mathcal{A}, \mathcal{V}]]]\right) e^{-\mathcal{B}(k(t))} \\
& -N^{-1 / 2} e^{\mathcal{B}(k(t))}\left([\mathcal{A}, \mathcal{V}]+N^{-1 / 2} \mathcal{V}\right) e^{-\mathcal{B}(k(t))}
\end{aligned}
$$

The function $\mu_{0}(t)$ and $h(\phi(t, x))$ appearing in (63a), (63b) are given below,

$$
\begin{aligned}
& \mu_{0}:=\int d x\left\{\frac{1}{2 i}\left(\phi \bar{\phi}_{t}-\bar{\phi} \phi_{t}\right)-|\nabla \phi|^{2}\right\}-\frac{1}{2} \int d x d y\left\{v_{N}(x-y)|\phi(x)|^{2}|\phi(y)|^{2}\right\} \\
& h(\phi(t, x)):=-\frac{1}{i} \partial_{t} \phi+\Delta \phi-\left(v_{N} *|\phi|^{2}\right) \phi .
\end{aligned}
$$

As we know, (63b) is set to be zero due to the Hartree equation for $\phi$, and (63c) which contains terms of order $O(1)$ becomes block diagonal by an appropriate choice for the evolution of $k$. We can compute the $O(1)$ term in (63c),

$$
(\underline{63 \mathrm{c}})=\mathcal{H}_{G}-\mathcal{I}\left(\begin{array}{cc}
w^{T} & \bar{f} \\
-f & -w
\end{array}\right)
$$

where

$$
\begin{aligned}
& f:=(\mathbf{S}(\operatorname{sh}(k))-\overline{\operatorname{ch}(k)} \circ m) \circ \operatorname{ch}(k)-(\mathbf{W}(\overline{\operatorname{ch}(k)})+\operatorname{sh}(k) \circ \bar{m}) \circ \operatorname{sh}(k) \\
& w:=(\mathbf{W}(\overline{\operatorname{ch}(k)})+\operatorname{sh}(k) \circ \bar{m}) \circ \overline{\operatorname{ch}(k)}-(\mathbf{S}(\operatorname{sh}(k))-\overline{\operatorname{ch}(k)} \circ m) \circ \overline{\operatorname{sh}(k)} .
\end{aligned}
$$

The evolution implies that $f=0$ thus

$$
\underline{(63 \mathrm{c})}=\mathcal{H}_{G}-\mathcal{I}\left(\begin{array}{cc}
w^{T} & 0 \\
0 & -w
\end{array}\right) \text {. }
$$

Multiplying $f$ on the right with $\overline{\operatorname{sh}(k)}$ and using the identities $\operatorname{ch}(k) \circ$ $\overline{\operatorname{sh}(k)}=\overline{\operatorname{sh}(k)} \circ \overline{\operatorname{ch}(k)}$ and $\operatorname{sh}(k) \circ \overline{\operatorname{sh}(k)}=(\overline{\operatorname{ch}(k)})^{2}-1$ we discover

$$
f \circ \overline{\operatorname{sh}(k)}+w \circ \overline{\operatorname{ch}(k)}=\mathbf{W}(\overline{\operatorname{ch}(k)})+\operatorname{sh}(k) \circ \bar{m} .
$$


Using the formula (see [21], lemma 3.1, stated in slightly different notation)

$$
\begin{aligned}
& \mathbf{W}(\overline{\operatorname{ch}(k)}) \circ \overline{\operatorname{ch}(k)}^{-1}+\overline{\operatorname{ch}(k)}^{-1} \circ \mathbf{W}(\overline{\operatorname{ch}(k)}) \\
& =\overline{\operatorname{ch}(k)}^{-1} \circ m \circ \overline{\operatorname{sh}(k)}-\operatorname{sh}(k) \circ \bar{m} \circ \overline{\operatorname{ch}(k)}^{-1}
\end{aligned}
$$

we get

$$
\begin{aligned}
& w(y, x)=\mathbf{W}(\overline{\operatorname{ch}(k)}) \circ(\overline{\operatorname{ch}(k)})^{-1}+\operatorname{sh}(k) \circ \bar{m} \circ(\overline{\operatorname{ch}(k)})^{-1}= \\
& \frac{1}{2}\left((\overline{\operatorname{ch}(k)})^{-1} \circ m \circ \overline{\operatorname{sh}(k)}+\operatorname{sh}(k) \circ \bar{m} \circ(\overline{\operatorname{ch}(k)})^{-1}\right)+\frac{1}{2}\left[\mathbf{W}(\overline{\operatorname{ch}(k)}),(\overline{\operatorname{ch}(k)})^{-1}\right] .
\end{aligned}
$$

If we write $w(y, x)$ for the above we have the quadratic term and a trace

$$
\begin{aligned}
\left(\underline{63 \mathrm{C})}=\mathcal{H}_{G}+\frac{1}{2}\left(\int d x d y\left\{w(x, y) \mathcal{D}_{y x}+w(y, x) \mathcal{D}_{x y}\right\}\right.\right. \\
\left.+\frac{1}{2} \operatorname{Tr}\left((\overline{\operatorname{ch}(k)})^{-1} \circ m \circ \overline{\operatorname{sh}(k)}+\operatorname{sh}(k) \circ \bar{m} \circ(\overline{\operatorname{ch}(k)})^{-1}\right)\right) .
\end{aligned}
$$

The error terms, which are order $O\left(N^{-1 / 2}\right)$ or higher, are all in the third line (63d) i.e. what we called $\mathcal{E}$,

$$
\mathcal{E}=e^{\mathcal{B}}\left([\mathcal{A}, \mathcal{V}]+N^{-1 / 2} \mathcal{V}\right) e^{-\mathcal{B}}
$$

Recall that

$$
\begin{aligned}
& {[\mathcal{A}, \mathcal{V}]=\int d x_{1} d x_{2}\left\{v_{N}\left(x_{1}-x_{2}\right)\left(\bar{\phi}\left(x_{2}\right) a_{x_{1}}^{*} \mathcal{Q}_{x_{1} x_{2}}+\phi\left(x_{2}\right) \mathcal{Q}_{x_{1} x_{2}}^{*} a_{x_{1}}\right)\right\}} \\
& \mathcal{V}=\frac{1}{2} \int d x_{1} d x_{2}\left\{v_{N}\left(x_{1}-x_{2}\right) \mathcal{Q}_{x_{1} x_{2}}^{*} \mathcal{Q}_{x_{1} x_{2}}\right\}
\end{aligned}
$$

and the transformation of $\left(a, a^{*}\right)$ is

$$
\begin{aligned}
e^{\mathcal{B}} a_{x} e^{-\mathcal{B}} & =\int\left(\operatorname{ch}(k)(y, x) a_{y}+\operatorname{sh}(k)(y, x) a_{y}^{*}\right) d y:=b_{x} \\
e^{\mathcal{B}} a_{x}^{*} e^{-\mathcal{B}} & =\int\left(\overline{\operatorname{sh}(k)}(y, x) a_{y}+\overline{\operatorname{ch}(k)}(y, x) a_{y}^{*}\right) d y:=b_{x}^{*} .
\end{aligned}
$$

One can see that $\left[b_{x}, b_{y}^{*}\right]=\delta(x-y)$. For the rest of this section, the argument of the "trigonometric" functions is always $k$, thus sh abbreviates $\operatorname{sh}(k), p$ stands for $p_{1}$, etc. Keeping in mind that $\overline{\operatorname{ch}}(y, x)=$ 
$\operatorname{ch}(x, y)$ and $\overline{\operatorname{sh}}(y, x)=\overline{\operatorname{sh}}(x, y), \operatorname{sh}(y, x)=\operatorname{sh}(x, y)$ we compute

$$
\begin{aligned}
& e^{\mathcal{B}} a_{x_{1}}^{*} a_{x_{2}}^{*} e^{-\mathcal{B}}=e^{\mathcal{B}} \mathcal{Q}_{x_{1} x_{2}}^{*} e^{-\mathcal{B}}= \\
& \int d y_{1} d y_{2}\left\{\overline{\operatorname{sh}}\left(y_{1}, x_{1}\right) \operatorname{ch}\left(x_{2}, y_{2}\right) \mathcal{D}_{y_{2} y_{1}}+\overline{\operatorname{ch}}\left(y_{1}, x_{1}\right) \overline{\operatorname{sh}}\left(x_{2}, y_{2}\right) \mathcal{D}_{y_{1} y_{2}}\right\}+ \\
& \int d y_{1} d y_{2}\left\{\overline{\operatorname{ch}}\left(y_{1}, x_{1}\right) \operatorname{ch}\left(x_{2}, y_{2}\right) \mathcal{Q}_{y_{1} y_{2}}^{*}+\overline{\operatorname{sh}}\left(y_{1}, x_{1}\right) \overline{\operatorname{sh}}\left(x_{2}, y_{2}\right) \mathcal{Q}_{y_{1} y_{2}}\right\} \\
& +(\overline{\operatorname{sh}} \circ \overline{\operatorname{ch}})\left(x_{1}, x_{2}\right) \\
& e^{\mathcal{B}} a_{x_{1}} a_{x_{2}} e^{-\mathcal{B}}=e^{\mathcal{B}} \mathcal{Q}_{x_{1} x_{2}} e^{-\mathcal{B}}= \\
& \int d y_{3} d y_{4}\left\{\operatorname{ch}\left(y_{3}, x_{1}\right) \operatorname{sh}\left(x_{2}, y_{4}\right) \mathcal{D}_{y_{4} y_{3}}+\operatorname{sh}\left(y_{3}, x_{1}\right) \overline{\operatorname{ch}}\left(x_{2}, y_{4}\right) \mathcal{D}_{y_{3} y_{4}}\right\}+ \\
& \int d y_{3} d y_{4}\left\{\operatorname{ch}\left(y_{3}, x_{1}\right) \overline{\operatorname{ch}}\left(x_{2}, y_{4}\right) \mathcal{Q}_{y_{3} y_{4}}+\operatorname{sh}\left(y_{3}, x_{1}\right) \operatorname{sh}\left(x_{2}, y_{4}\right) \mathcal{Q}_{y_{3} y_{4}}^{*}\right\} \\
& +(\overline{\operatorname{ch}} \circ \operatorname{sh})\left(x_{1}, x_{2}\right) .
\end{aligned}
$$

Let us look first at the all the terms that come from the quartic term $\mathcal{V}$. Since $e^{\mathcal{B}} \mathcal{V} e^{-\mathcal{B}}|0\rangle$ we are interested only in terms that do not anihilate the vacuum. This implies that we keep $\mathcal{Q}_{y_{3} y_{4}}^{*}$ from (67b) with everything in (67a) as well as $\mathcal{Q}_{y_{1} y_{2}}^{*}$ from (67a with the last term in (67b) and the product of the two last terms in (67a), 67b).

Below is the list of all the terms in $\frac{1}{N} e^{\mathcal{B}} \mathcal{V} e^{-\mathcal{B}}$ (which do not annihilate $|0\rangle)$ in raw form,

$$
\begin{aligned}
& \frac{1}{2 N} \quad \int d x_{1} d x_{2} d y_{1} d y_{2} d y_{3} d y_{4}\{ \\
& \overline{\operatorname{sh}}\left(y_{1}, x_{1}\right) \operatorname{ch}\left(x_{2}, y_{2}\right) v_{N}\left(x_{1}-x_{2}\right) \operatorname{sh}\left(y_{3}, x_{1}\right) \operatorname{sh}\left(x_{2}, y_{4}\right) \mathcal{D}_{y_{2} y_{1}} \mathcal{Q}_{y_{3} y_{4}}^{*}+ \\
& \overline{\operatorname{ch}}\left(y_{1}, x_{1}\right) \overline{\operatorname{sh}}\left(x_{2}, y_{2}\right) v_{N}\left(x_{1}-x_{2}\right) \operatorname{sh}\left(y_{3}, x_{1}\right) \operatorname{sh}\left(x_{2}, y_{4}\right) \mathcal{D}_{y_{1} y_{2}} \mathcal{Q}_{y_{3} y_{4}}^{*}+ \\
& \overline{\operatorname{ch}}\left(y_{1}, x_{1}\right) \operatorname{ch}\left(x_{2}, y_{2}\right) v_{N}\left(x_{1}-x_{2}\right) \operatorname{sh}\left(y_{3}, x_{1}\right) \operatorname{sh}\left(x_{2}, y_{4}\right) \mathcal{Q}_{y_{1} y_{2}}^{*} \mathcal{Q}_{y_{3} y_{4}}^{*}+ \\
& \left.\overline{\operatorname{sh}}\left(y_{1}, x_{1}\right) \overline{\operatorname{sh}}\left(x_{2}, y_{2}\right) v_{N}\left(x_{1}-x_{2}\right) \operatorname{sh}\left(y_{3}, x_{1}\right) \operatorname{sh}\left(x_{2}, y_{4}\right) \mathcal{Q}_{y_{1} y_{2}} \mathcal{Q}_{y_{3} y_{4}}^{*}\right\} \\
& +\frac{1}{2 N} \int d x_{1} d x_{2} d y_{1} d y_{2}\{ \\
& (\overline{\operatorname{sh}} \circ \overline{\mathrm{ch}})\left(x_{1}, x_{2}\right) v_{N}\left(x_{1}-x_{2}\right) \operatorname{sh}\left(y_{1}, x_{1}\right) \operatorname{sh}\left(x_{2}, y_{2}\right) \mathcal{Q}_{y_{1} y_{2}}^{*}+ \\
& \left.\overline{\operatorname{ch}}\left(y_{1}, x_{1}\right) \operatorname{ch}\left(x_{2}, y_{2}\right) v_{N}\left(x_{1}-x_{2}\right)(\overline{\operatorname{ch}} \circ \operatorname{sh})\left(x_{1}, x_{2}\right) \mathcal{Q}_{y_{1} y_{2}}^{*}\right\} \\
& +\frac{1}{2 N} \quad \int d x_{1} d x_{2} \\
& (\overline{\operatorname{sh}} \circ \overline{\mathrm{ch}})\left(x_{1}, x_{2}\right) v_{N}\left(x_{1}-x_{2}\right)(\overline{\mathrm{ch}} \circ \mathrm{sh})\left(x_{1}, x_{2}\right) \text {. }
\end{aligned}
$$


Next let us look at the terms that come from the cubic term, namely the expression $e^{\mathcal{B}}[\mathcal{A}, \mathcal{V}] e^{-\mathcal{B}}|0\rangle$. They come in two sets. First from the term $b_{x_{1}}^{*}\left(b_{x_{1}} b_{x_{2}}\right)$ there are three terms, two coming from the product of $\mathcal{Q}^{*}$ from (67b) with (66b) and one coming from $a^{*}$ in (66b) with the constant term in (67b). The are listed below

$$
\begin{aligned}
& N^{-1 / 2} \int d x_{1} d x_{2} d y_{1} d y_{2} d y_{3}\{ \\
& \overline{\operatorname{sh}}\left(y_{1}, x_{1}\right) v_{N}\left(x_{1}-x_{2}\right) \bar{\phi}\left(x_{2}\right) \operatorname{sh}\left(y_{2}, x_{1}\right) \operatorname{sh}\left(x_{2}, y_{3}\right) a_{y_{1}} \mathcal{Q}_{y_{2} y_{3}}^{*}+ \\
& \left.\overline{\operatorname{ch}}\left(y_{1}, x_{1}\right) v_{N}\left(x_{1}-x_{2}\right) \bar{\phi}\left(x_{2}\right) \operatorname{sh}\left(y_{2}, x_{1}\right) \operatorname{sh}\left(x_{2}, y_{3}\right) a_{y_{1}}^{*} \mathcal{Q}_{y_{2} y_{3}}^{*}\right\}+ \\
& N^{-1 / 2} \int d x_{1} d x_{2} d y_{1} \overline{\operatorname{ch}}\left(y_{1}, x_{1}\right) v_{N}\left(x_{1}-x_{2}\right) \bar{\phi}\left(x_{2}\right)(\overline{\operatorname{ch}} \circ \operatorname{sh})\left(x_{1}, x_{2}\right) a_{y_{1}}^{*}
\end{aligned}
$$

The second set comes from $\left(b_{x_{1}}^{*} b_{x_{2}}^{*}\right) b_{x_{1}}$ and gives five terms, namely all terms appearing in (67a) multiplied with $a^{*}$ in (66b). They are listed below,

$$
\begin{aligned}
& N^{-1 / 2} \int d x_{1} d x_{2} d y_{1} d y_{2} d y_{3}\{ \\
& \overline{\operatorname{sh}}\left(y_{1}, x_{1}\right) \operatorname{ch}\left(x_{2}, y_{2}\right) v_{N}\left(x_{1}-x_{2}\right) \phi\left(x_{2}\right) \operatorname{sh}\left(y_{3}, x_{1}\right) \mathcal{D}_{y_{2} y_{1}} a_{y_{3}}^{*}+ \\
& \overline{\operatorname{ch}}\left(y_{1}, x_{1}\right) \overline{\operatorname{sh}}\left(x_{2}, y_{2}\right) v_{N}\left(x_{1}-x_{2}\right) \phi\left(x_{2}\right) \operatorname{sh}\left(y_{3}, x_{1}\right) \mathcal{D}_{y_{1} y_{2}} a_{y_{3}}^{*}+ \\
& \overline{\operatorname{ch}}\left(y_{1}, x_{1}\right) \operatorname{ch}\left(x_{2}, y_{2}\right) v_{N}\left(x_{1}-x_{2}\right) \phi\left(x_{2}\right) \operatorname{sh}\left(y_{3}, x_{1}\right) \mathcal{Q}_{y_{1} y_{2}}^{*} a_{y_{3}}^{*}+ \\
& \left.\overline{\operatorname{sh}}\left(y_{1}, x_{1}\right) \overline{\operatorname{sh}}\left(x_{2}, y_{2}\right) v_{N}\left(x_{1}-x_{2}\right) \phi\left(x_{2}\right) \operatorname{sh}\left(y_{3}, x_{1}\right) \mathcal{Q}_{y_{1} y_{2}} a_{y_{3}}^{*}\right\}+ \\
& N^{-1 / 2} \int d x_{1} d x_{2} d y_{1}(\overline{\operatorname{sh}} \circ \overline{\operatorname{ch}})\left(x_{1}, x_{2}\right) v_{N}\left(x_{1}-x_{2}\right) \phi\left(x_{2}\right) \operatorname{sh}\left(y_{1}, x_{1}\right) a_{y_{1}}^{*}
\end{aligned}
$$

Some of the terms can be reduced to lower order by commuting $a$ with $a^{*}$ whenever they appear together in a product. The term in (70d) applied to $|0\rangle$ gives zero. Irreducible terms are those appearing in (68c) which is fourth order, as well as the terms appearing in (69b) and (70c) which are cubic. The quartic irreducible term in (68c) can 
be writen, if we write $\operatorname{ch}(x, y)=\delta(x-y)+p(x, y)$ first, as follows,

$$
\begin{aligned}
& N^{-1}(1 / 2)\left\{v_{N}\left(y_{1}-y_{2}\right) \operatorname{sh}\left(y_{3}, y_{1}\right) \operatorname{sh}\left(y_{2}, y_{4}\right)+\right. \\
& \int d x\left\{\bar{p}\left(y_{2}, x\right) v_{N}\left(y_{1}-x\right) \operatorname{sh}\left(x, y_{4}\right)\right\} \operatorname{sh}\left(y_{3}, y_{1}\right)+ \\
& \int d x\left\{\bar{p}\left(y_{1}, x\right) v_{N}\left(x-y_{2}\right) \operatorname{sh}\left(y_{3}, x\right)\right\} \operatorname{sh}\left(y_{2}, y_{4}\right)+ \\
& \left.\int d x_{1} d x_{2}\left\{\bar{p}\left(y_{1}, x_{1}\right) p\left(x_{2}, y_{2}\right) v_{N}\left(x_{1}-x_{2}\right) \operatorname{sh}\left(y_{3}, x_{1}\right) \operatorname{sh}\left(x_{2}, y_{4}\right)\right\}\right\} .
\end{aligned}
$$

The rest of the quartic terms can be reduced to either quadratic or zero order terms. For example if we look at (68b) we can write, $\mathcal{D}_{y_{1} y_{2}} \mathcal{Q}_{y_{3} y_{4}}^{*}=$ $\delta\left(y_{2}-y_{4}\right) \mathcal{Q}_{y_{1} y_{4}}^{*}+\delta\left(y_{2}-y_{4}\right) \mathcal{Q}_{y_{1} y_{3}}^{*}$ (modulo terms which annihilate $\left.|0\rangle\right)$ and similarly for (68a). Below is a list of all quadatic terms,

$$
\begin{aligned}
& (1 / 2 N) \int d x_{1} d x_{2} \quad\{ \\
& \overline{\operatorname{ch}}\left(y_{1}, x_{2}\right) \operatorname{sh}\left(x_{2}, y_{2}\right)(\overline{\operatorname{sh}} \circ \operatorname{sh})\left(x_{1}, x_{1}\right) v_{N}\left(x_{1}-x_{2}\right)+ \\
& \overline{\operatorname{ch}}\left(y_{1}, x_{2}\right) \operatorname{sh}\left(x_{1}, y_{2}\right)(\overline{\operatorname{sh}} \circ \operatorname{sh})\left(x_{1}, x_{2}\right) v_{N}\left(x_{1}-x_{2}\right)+ \\
& \overline{\operatorname{ch}}\left(y_{1}, x_{1}\right) \operatorname{sh}\left(x_{2}, y_{2}\right)(\operatorname{sh} \circ \overline{\operatorname{sh}})\left(x_{1}, x_{2}\right) v_{N}\left(x_{1}-x_{2}\right)+ \\
& \overline{\operatorname{ch}}\left(y_{1}, x_{1}\right) \operatorname{sh}\left(x_{1}, y_{2}\right)(\overline{\operatorname{sh}} \circ \overline{\operatorname{sh}})\left(x_{2}, x_{2}\right) v_{N}\left(x_{1}-x_{2}\right)+ \\
& \operatorname{sh}\left(y_{1}, x_{1}\right) \operatorname{sh}\left(x_{2}, y_{2}\right)(\overline{\operatorname{sh}} \circ \overline{\operatorname{ch}})\left(x_{1}, x_{2}\right) v_{N}\left(x_{1}-x_{2}\right)+ \\
& \left.\overline{\operatorname{ch}}\left(y_{1}, x_{1}\right) \operatorname{ch}\left(x_{2}, y_{2}\right)(\overline{\operatorname{ch}} \circ \operatorname{sh})\left(x_{1}, x_{2}\right) v_{N}\left(x_{1}-x_{2}\right)\right\}
\end{aligned}
$$

In addition (68d) and (68g) together with the trace in (64) will supply a zero order term,

$$
\begin{aligned}
\mu_{1}= & -\frac{1}{4} \operatorname{Tr}\left((\overline{\operatorname{ch}(k)})^{-1} \circ m \circ \overline{\operatorname{sh}(k)}+\operatorname{sh}(k) \circ \bar{m} \circ(\overline{\operatorname{ch}(k)})^{-1}\right) \\
& +\frac{1}{2 N} \int d x_{1} d x_{2}\left\{(\operatorname{sh} \circ \overline{\operatorname{sh}})\left(x_{1}, x_{2}\right) v_{N}\left(x_{1}-x_{2}\right)(\overline{\operatorname{sh}} \circ \operatorname{sh})\left(x_{1}, x_{2}\right)+\right. \\
& (\operatorname{sh} \circ \overline{\operatorname{sh}})\left(x_{1}, x_{1}\right) v_{N}\left(x_{1}-x_{2}\right)(\overline{\operatorname{sh}} \circ \operatorname{sh})\left(x_{2}, x_{2}\right)+ \\
& \left.(\overline{\operatorname{sh}} \circ \overline{\operatorname{ch}})\left(x_{1}, x_{2}\right) v_{N}\left(x_{1}-x_{2}\right)(\overline{\operatorname{ch}} \circ \operatorname{sh})\left(x_{1}, x_{2}\right)\right\}
\end{aligned}
$$

This term can be absorbed as a phase factor in the evolution. The cubic irreducible terms are those appearing in (69b), (170c). Writing 
$\overline{\operatorname{ch}}(y, x)=\delta(y-x)+\bar{p}(y, x)$ we can express them in the manner below

$$
\begin{aligned}
& N^{-1 / 2}\left\{v_{N}\left(y_{1}-y_{2}\right) \phi\left(y_{2}\right) \operatorname{sh}\left(y_{3}, y_{1}\right) \quad+\right. \\
& \int d x\left\{v_{N}\left(y_{1}-x\right) \bar{\phi}(x) \operatorname{sh}\left(x, y_{3}\right)\right\} \operatorname{sh}\left(y_{2}, y_{1}\right)+ \\
& \int d x\left\{\bar{p}\left(y_{1}, x\right) v_{N}\left(x-y_{2}\right) \operatorname{sh}\left(y_{3}, x\right)\right\} \phi\left(y_{2}\right)+ \\
& \int d x\left\{\bar{p}\left(y_{2}, x\right) v_{N}\left(y_{1}-x\right) \phi(x)\right\} \operatorname{sh}\left(y_{3}, y_{1}\right)+ \\
& \int d x_{1} d x_{2}\left\{\bar{p}\left(y_{1}, x_{1}\right) v_{N}\left(x_{1}-x_{2}\right) \bar{\phi}\left(x_{2}\right) \operatorname{sh}\left(y_{2}, x_{1}\right) \operatorname{sh}\left(x_{2}, y_{3}\right)\right\}+ \\
& \left.\int d x_{1} d x_{2}\left\{\bar{p}\left(y_{1}, x_{1}\right) p\left(x_{2}, y_{2}\right) v_{N}\left(x_{1}-x_{2}\right) \phi\left(x_{2}\right) \operatorname{sh}\left(y_{3}, x_{1}\right)\right\}\right\} .
\end{aligned}
$$

The rest can be reduced to linear or eliminated, for example (70d) can be eliminated. For the cubic terms in the first set. From the commutator relation $a_{y_{1}} \mathcal{Q}_{y_{2} y_{3}}^{*}=\delta\left(y_{1}-y_{2}\right) a_{y_{3}}^{*}+\delta\left(y_{1}-y_{3}\right) a_{y_{2}}^{*}$ (modulo a terms which kill $|0\rangle)$ we reduce them to linear and here is the list,

$$
\begin{aligned}
& N^{-1 / 2} \int d x_{1} d x_{2}\{ \\
& \operatorname{sh}\left(y_{1}, x_{2}\right)(\overline{\operatorname{sh}} \circ \operatorname{sh})\left(x_{1}, x_{1}\right) \bar{\phi}\left(x_{2}\right) v_{N}\left(x_{1}-x_{2}\right)+ \\
& \operatorname{sh}\left(y_{1}, x_{1}\right)(\overline{\operatorname{sh}} \circ \operatorname{sh})\left(x_{1}, x_{2}\right) \bar{\phi}\left(x_{2}\right) v_{N}\left(x_{1}-x_{2}\right)+ \\
& \left.\overline{\operatorname{ch}}\left(y_{1}, x_{1}\right)(\overline{\operatorname{ch}} \circ \operatorname{sh})\left(x_{1}, x_{2}\right) \bar{\phi}\left(x_{2}\right) v_{N}\left(x_{1}-x_{2}\right)\right\} .
\end{aligned}
$$

From the cubic terms in the second list we have the commutators $\mathcal{D}_{y_{1} y_{2}} a_{y_{3}}^{*}=\delta\left(y_{2}-y_{3}\right) a_{y_{1}}^{*}$ and $\mathcal{D}_{y_{2} y_{1}} a_{y_{3}}^{*}=\delta\left(y_{1}-y_{3}\right) a_{y_{2}}^{*}$ (modulo $a$ terms) hence from (70a), (70b) as well as from (70e) we obtain

$$
\begin{aligned}
& N^{-1 / 2} \int d x_{1} d x_{2}\{ \\
& \overline{\operatorname{ch}}\left(y_{1}, x_{1}\right)(\operatorname{sh} \circ \overline{\operatorname{sh}})\left(x_{1}, x_{2}\right) \phi\left(x_{2}\right) v_{N}\left(x_{1}-x_{2}\right)+ \\
& \overline{\operatorname{ch}}\left(y_{2}, x_{2}\right)(\operatorname{sh} \circ \overline{\operatorname{sh}})\left(x_{1}, x_{1}\right) \phi\left(x_{2}\right) v_{N}\left(x_{1}-x_{2}\right)+ \\
& \left.\operatorname{sh}\left(y_{1}, x_{1}\right)(\overline{\operatorname{sh}} \circ \overline{\operatorname{ch}})\left(x_{1}, x_{2}\right) \phi\left(x_{2}\right) v_{N}\left(x_{1}-x_{2}\right)\right\} .
\end{aligned}
$$

\section{Estimates FOR THE ERROR TERMS}

In this section we prove 
Proposition 6.1. The following estimates for the error terms holds:

$$
\begin{aligned}
& N^{-1 / 2} \| e^{\mathcal{B}}[\mathcal{A}, \mathcal{V}] e^{-\mathcal{B}}|0\rangle \|_{\mathbb{F}} \leq N^{\frac{3 \beta-1}{2}} \frac{\log ^{3}(1+t)}{t^{3 / 2}} \\
& N^{-1} \| e^{\mathcal{B}} \mathcal{V} e^{-\mathcal{B}}|0\rangle \|_{\mathbb{F}} \leq C N^{3 \beta-1} \log ^{4}(1+t)
\end{aligned}
$$

Proof. All estimates are straightforward, based on Corollary (4.2) and Lemma (3.4), and we only include a few typical ones from each category.

Estimate for (71a):

$$
\begin{aligned}
& N^{-1}\left\|v_{N}\left(y_{1}-y_{2}\right) \operatorname{sh}(k)\left(y_{3}, y_{1}\right) \operatorname{sh}(k)\left(y_{2}, y_{4}\right)\right\|_{L^{2}\left(d y_{1} d y_{2} d y_{3} d y_{4}\right)} \\
& \leq N^{-1}\left\|v_{N}\right\|_{L^{\infty}}\|\operatorname{sh}(k)\|_{L^{2}}^{2} \leq C N^{3 \beta-1} \log ^{2}(1+t)
\end{aligned}
$$

Estimate for (71d):

$$
\begin{aligned}
& N^{-1}\left\|\int d x_{1} d x_{2} \bar{p}_{1}\left(y_{1}, x_{1}\right) p_{1}\left(x_{2}, y_{2}\right) v_{N}\left(x_{1}-x_{2}\right) \operatorname{sh}\left(y_{3}, x_{1}\right) \operatorname{sh}\left(x_{2}, y_{4}\right)\right\|_{L^{2}\left(d y_{1} d y_{2} d y_{3} d y_{4}\right)} \\
& \leq N^{-1}\left\|v_{N}\right\|_{L^{\infty}}\|\operatorname{sh}(k)\|_{L^{2}}^{2}\left\|p_{1}\right\|_{L^{2}}^{2} \leq C N^{3 \beta-1} \log ^{4}(1+t)
\end{aligned}
$$

Estimate for (72f), keeping only the $\delta$ contribution in $\operatorname{ch}(k)$ :

$$
N^{-1}\left\|\operatorname{sh}(k)\left(y_{1}, y_{2}\right) v_{N}\left(y_{1}-y_{2}\right)\right\|_{L^{2}\left(d y_{1} d y_{2}\right)} \leq\left\|v_{N}\right\|_{L^{\infty}}\|\operatorname{sh}(k)\|_{L^{2}} \leq C N^{3 \beta-1} \log (1+t)
$$

Estimate for (74a), :

$$
\begin{aligned}
& N^{-1 / 2}\left\|v_{N}\left(y_{1}-y_{2}\right) \phi\left(y_{2}\right) \operatorname{sh}(k)\left(y_{3}, y_{1}\right)\right\|_{L^{2}\left(d y_{1} d y_{2} d y_{3}\right)} \\
& \leq\|\phi\|_{L^{\infty}}\left\|v_{N}\right\|_{L^{2}}\|\operatorname{sh}(k)\|_{L^{2}} \leq C N^{\frac{3 \beta-1}{2}} \frac{\log (1+t)}{t^{3 / 2}}
\end{aligned}
$$

Estimates for (76b):

$$
\begin{aligned}
& N^{-1 / 2}\left\|\int d x_{1} d x_{2} p_{2}\left(y_{2}, x_{2}\right)(\operatorname{sh}(k) \circ \overline{\operatorname{sh}(k)})\left(x_{1}, x_{1}\right) \phi\left(x_{2}\right) v_{N}\left(x_{1}-x_{2}\right)\right\|_{L^{2}\left(d y_{2}\right)} \\
& \leq N^{-1 / 2}\left\|p_{2}\right\|_{L^{2}}\|\operatorname{sh}(k) \circ \overline{\operatorname{sh}(k)}(x, x)\|_{L^{1}(d x)}\|\phi\|_{L^{\infty}}\left\|v_{N}\right\|_{L^{2}} \\
& \leq N^{-1 / 2}\left\|p_{2}\right\|_{L^{2}}\|\operatorname{sh}(k)\|_{L^{2}}^{2}\|\phi\|_{L^{\infty}}\left\|v_{N}\right\|_{L^{2}} \\
& \leq C N^{\frac{3 \beta-1}{2}} \frac{\log ^{3}(1+t)}{t^{3 / 2}}
\end{aligned}
$$

\section{Appendix}

The purpose of this appendix is to make certain connections with our previous work [20], 21] and to provide some useful information. We would like to explain first the Lie algebra isomorphism which is crucial in our work. This was explained in [20] but we include it here 
for completeness and for the convenience of the reader. Let us define first

$$
\mathcal{A}_{x}:=\left(\begin{array}{l}
a_{x} \\
a_{x}^{*}
\end{array}\right) \quad \text { and } \quad \mathbf{f}(x):=\left(\begin{array}{l}
f_{1}(x) \\
f_{2}(x)
\end{array}\right) \quad \text { and } \quad J=\left(\begin{array}{cc}
0 & -1 \\
1 & 0
\end{array}\right)
$$

and using these form

$$
\mathcal{A}(\mathbf{f}):=\int d x\left\{\mathcal{A}_{x}^{T} \mathbf{f}(x)\right\}=\int d x\left\{f_{1}(x) a_{x}+f_{2}(x) a_{x}^{*}\right\} .
$$

It is straightforward to check the commutation,

$$
\begin{aligned}
{[\mathcal{A}(\mathbf{f}), \mathcal{A}(\mathbf{g})] } & =\int d x\left\{f_{1}(x) g_{2}(x)-f_{2}(x) g_{1}(x)\right\} \\
& =-\int d x d y\left\{\mathbf{f}^{T}(x) \delta(x-y) J \mathbf{g}(y)\right\}
\end{aligned}
$$

For $L^{2}(d x d y)$ kernel functions $d(t, x, y)$ and $k(t, x, y), l(t, x, y)$ such that $k$ and $l$ are symmetric in the $(x, y)$ variables form the symplectic matrix

$$
S(d, k, l):=\left(\begin{array}{cc}
d & k \\
l & -d^{T}
\end{array}\right) .
$$

We will write $S(x, y)$ when convenient, suppressing the $t$ dependence. Next we define the map $\mathcal{I}: \operatorname{sp}(\mathbb{C}) \mapsto$ Quad from the space of complex, $L^{2}$ symplectic matrices to quadratic expressions in $\left(a, a^{*}\right)$ as follows :

$$
\mathcal{I}(S):=-\frac{1}{2} \int d x d y\left\{\mathcal{A}_{x}^{T} S(x, y) J \mathcal{A}_{y}\right\}
$$

Theorem 7.1. Let $\mathbf{f}(x)$ a vector function and $\mathcal{A}(\mathbf{f})$ the corresponding expression (77). Then the following commutations relations hold

$$
\begin{aligned}
& {[\mathcal{I}(S), \mathcal{A}(\mathbf{f})]=\mathcal{A}(S \circ \mathbf{f})} \\
& e^{\mathcal{I}(S)} \mathcal{A}(\mathbf{f}) e^{-\mathcal{I}(S)}=\mathcal{A}\left(e^{S} \circ \mathbf{f}\right)
\end{aligned}
$$

where (for example) $S \circ \mathbf{f}=\int d x\{S(x, y) \mathbf{f}(y)\}$ etc. Formula (81b) holds for any complex symplectic $S \in \operatorname{sp}_{\mathrm{c}}(\mathbb{R})=\left(\mathrm{C}^{\mathrm{T}}\right)^{-1} \mathrm{sp}(\mathbb{R}) \mathrm{C}^{\mathrm{T}}$ where $C$ is the change-of-basis matrix

$$
C=\frac{1}{\sqrt{2}}\left(\begin{array}{cc}
I & -i I \\
I & i I
\end{array}\right)
$$

and $\operatorname{sp}(\mathbb{R})$ is the Lie algebra of real symplectic matrices. This condition insures that $e^{S}$ is unitary. 
Proof. The commutation relation in (81a) can be easily checked, but we point that it follows from (78). For any rank one quadratics we have using (78)

$$
[\mathcal{A}(\mathbf{f}) \mathcal{A}(\mathbf{g}), \mathcal{A}(\mathbf{h})]=-\mathcal{A}\left(\left(\mathbf{g f}^{T}+\mathbf{f g}^{T}\right) \circ J \mathbf{h}\right)
$$

Thus for any $R$ we have

$$
\left[\int d x d y\left\{\mathcal{A}_{x}^{T} R(x, y) \mathcal{A}_{y}\right\}, \mathcal{A}(\mathbf{f})\right]=-\mathcal{A}\left(\left(R+R^{T}\right) \circ J \mathbf{f}\right)
$$

Now specialize to $R=(1 / 2) S J$ with $S \in$ sp and use $S^{T}=J S J$ to complete the proof.

For the second formula, introduce a complex parameter $t$ and take $|\psi\rangle$ a Fock space vector with finitely many non-zero components. It is trivial to check, using (81a), that all $t$ derivatives of $e^{t \mathcal{I}(S)} \mathcal{A}(\mathbf{f}) e^{-t \mathcal{I}(S)}|\psi\rangle$ and $\mathcal{A}\left(e^{t S} \circ \mathbf{f}\right)|\psi\rangle$ agree when $t=0$, and both the left hand side and right hand side are analytic if $|t|$ is sufficiently small. Thus they agree for all $t$ complex, sufficiently small. To take $t$ large, we have to restrict ourselves to real $t$ and use the group properties of the unitary family $e^{t S}$.

A more formal (but convincing) "proof" follows from

$$
e^{\mathcal{I}(S)} \mathcal{C} e^{-\mathcal{I}(S)}=\mathcal{C}+[\mathcal{I}(S), \mathcal{C}]+\frac{1}{2 !}[\mathcal{I}(S),[\mathcal{I}(S), \mathcal{C}]]+\ldots
$$

Theorem 7.2. The map $\mathcal{I}: \operatorname{sp}(\mathbb{C}) \mapsto$ Quad defined in (180) is a Lie algebra isomorphism. Moreover for $S, R \in \mathrm{sp}_{\mathrm{c}}(\mathbb{R})$ we have the formulas

$$
\begin{aligned}
& \mathcal{I}\left(\left(\partial_{t} e^{S}\right) \circ e^{-S}\right)=\left(\partial_{t} e^{\mathcal{I}(S)}\right) e^{-\mathcal{I}(S)} \\
& \mathcal{I}\left(e^{S} \circ R \circ e^{-S}\right)=e^{\mathcal{I}(S)} \mathcal{I}(R) e^{-\mathcal{I}(S)} .
\end{aligned}
$$

Proof. We point out that (81b) implies (82b), at least when $R$ is rank one, $R:=\mathbf{f}(x) \mathbf{g}^{T}(y)$. Notice that (81b) can be written

$$
e^{\mathcal{I}(S)} \int d x\left\{\mathbf{f}^{T}(x) \mathcal{A}_{x}\right\} e^{-\mathcal{I}(S)}=\int d x d y\left\{\mathbf{f}^{T}(x) e^{S^{T}} \mathcal{A}_{y}\right\}
$$


from which we have

$$
\begin{aligned}
& e^{\mathcal{I}(S)} \int d x d y\left\{\mathcal{A}_{x}^{T} \mathbf{f}(x) \mathbf{g}^{T}(y) J \mathcal{A}_{y}\right\} e^{-\mathcal{I}(S)} \\
& =e^{\mathcal{I}(S)} \mathcal{A}(\mathbf{f}) e^{-\mathcal{I}(S)} e^{\mathcal{I}(S)} \int d y\left\{\mathbf{g}^{T}(y) J \mathcal{A}_{y}\right\} e^{-\mathcal{I}(S)} \\
& =\mathcal{A}\left(e^{S} \mathbf{f}\right) \int d y d z\left\{\mathbf{g}^{T}(y) J e^{J S J} \mathcal{A}_{z}\right\} \\
& =\int d x d y\left\{\mathcal{A}_{x}^{T} e^{S} R e^{-S} J \mathcal{A}_{y}\right\}
\end{aligned}
$$

since $S^{T}=J S J$ and $J e^{J S J}=e^{-S} J$.

A direct proof of the Lie algebra isomorphism follows from the following elementary computations. Define first

$$
\mathcal{Q}_{x y}:=a_{x} a_{y} \quad \text { and } \quad \mathcal{Q}_{x y}^{*}:=a_{x}^{*} a_{y}^{*} \quad \text { and } \quad \mathcal{N}_{x y}:=\frac{1}{2}\left(a_{x} a_{y}^{*}+a_{y}^{*} a_{x}\right) .
$$

It is straightforward to verify,

$$
\begin{aligned}
& {\left[\mathcal{Q}_{x_{1} x_{2}}, \mathcal{Q}_{y_{1} y_{2}}^{*}\right]=\delta\left(x_{1}-y_{1}\right) \mathcal{N}_{x_{2} y_{2}}+\delta\left(x_{1}-y_{2}\right) \mathcal{N}_{x_{2} y_{1}}+\delta\left(x_{2}-y_{1}\right) \mathcal{N}_{x_{1} y_{2}}+\delta\left(x_{2}-y_{2}\right) \mathcal{N}_{x_{1} y_{1}}} \\
& {\left[\mathcal{Q}_{x_{1} x_{2}}, \mathcal{N}_{y_{1} y_{2}}\right]=\delta\left(x_{1}-y_{2}\right) \mathcal{Q}_{x_{2} y_{1}}+\delta\left(x_{2}-y_{2}\right) \mathcal{Q}_{x_{1} y_{1}}} \\
& {\left[\mathcal{N}_{x_{1} x_{2}}, \mathcal{Q}_{y_{1} y_{2}}^{*}\right]=\delta\left(x_{1}-y_{1}\right) \mathcal{Q}_{x_{2} y_{2}}^{*}+\delta\left(x_{1}-y_{2}\right) \mathcal{Q}_{x_{2} y_{1}}^{*}} \\
& {\left[\mathcal{N}_{x_{1} x_{2}}, \mathcal{N}_{y_{1} y_{2}}\right]=\delta\left(x_{1}-y_{2}\right) \mathcal{N}_{y_{1} x_{2}}-\delta\left(x_{2}-y_{1}\right) \mathcal{N}_{x_{1} y_{2}}}
\end{aligned}
$$

and using these we can directly verify that

$$
\begin{aligned}
& \frac{1}{2}\left[\int d x_{1} d x_{2}\left\{k\left(x_{1}, x_{2}\right) \mathcal{Q}_{x_{1} x_{2}}\right\},-\int d y_{1} d y_{2}\left\{l\left(y_{1}, y_{2}\right) \mathcal{Q}_{y_{1} y_{2}}^{*}\right\}\right] \\
& =-\int d x d y\left\{(k \circ l)(x, y) \mathcal{N}_{x y}\right\}
\end{aligned}
$$

which corresponds to the relation

$$
\left[\left(\begin{array}{ll}
0 & k \\
0 & 0
\end{array}\right),\left(\begin{array}{ll}
0 & 0 \\
l & 0
\end{array}\right)\right]=\left(\begin{array}{cc}
k \circ l & 0 \\
0 & -l \circ k
\end{array}\right) .
$$

The other cases are checked in a similar manner.

To prove (82a), expand both the left and right-hand side as

$$
\begin{aligned}
& \mathcal{I}\left(\left(\partial_{t} e^{S}\right) e^{-S}\right)=\mathcal{I}\left(\dot{S}+\frac{1}{2}[S, \dot{S}]+\ldots\right) \\
& =\dot{\mathcal{I}}(S)+\frac{1}{2}[\mathcal{I}(S), \dot{\mathcal{I}}(S)]+\ldots=\left(\partial_{t} e^{\mathcal{I}(S)}\right) e^{-\mathcal{I}(S)} .
\end{aligned}
$$

The proof of $(82 \mathrm{~b})$ is along the same lines. The proofs can be made rigorous by an analyticity argument on the dense subset of vectors with finitely many non-zero components. 
The second part of this appendix is devoted to the connection between the present equations for the pair excitations (17b), (17c) with the evolution equation derived in [20] and [21], the idea being that the nonlinear equation derived in these references turns out to be linear when expressed in new coordinates. The connection however is not entirely obvious. From the identities,

$$
\begin{aligned}
e^{2 K} & =\left(\begin{array}{ll}
\operatorname{ch}(2 k) & \overline{\operatorname{sh}(2 k)} \\
\operatorname{sh}(2 k) & \overline{\operatorname{ch}(2 k)}
\end{array}\right) \\
& =\left(\begin{array}{ll}
\operatorname{ch}(k) \circ \operatorname{ch}(k)+\overline{\operatorname{sh}(k)} \circ \operatorname{sh}(k) & \operatorname{ch}(k) \circ \overline{\operatorname{sh}(k)}+\overline{\operatorname{sh}(k)} \circ \overline{\operatorname{ch}(k)} \\
\operatorname{sh}(k) \circ \operatorname{ch}(k)+\overline{\operatorname{ch}(k)} \circ \overline{\operatorname{sh}(k)} & \operatorname{sh}(k) \circ \overline{\operatorname{sh}(k)}+\overline{\operatorname{ch}(k)} \circ \overline{\operatorname{ch}(k)}
\end{array}\right) \\
& =\left(\begin{array}{cc}
2 \overline{\operatorname{sh}(k)} \circ \overline{\operatorname{sh}(k)+1} & 2 \overline{\operatorname{sh}(k)} \circ \overline{\operatorname{ch}(k)} \\
2 \overline{\operatorname{ch}(k)} \circ \operatorname{sh}(k) & 2 \operatorname{sh}(k) \circ \overline{\operatorname{sh}(k)}+1
\end{array}\right)
\end{aligned}
$$

and

$$
I=\left(\begin{array}{ll}
\operatorname{ch}(k) \circ \operatorname{ch}(k)-\overline{\operatorname{sh}(k)} \circ \operatorname{sh}(k) & -\operatorname{ch}(k) \circ \overline{\operatorname{sh}(k)}+\overline{\operatorname{sh}(k)} \circ \overline{\operatorname{ch}(k)} \\
\operatorname{sh}(k) \circ \operatorname{ch}(k)-\overline{\operatorname{ch}(k)} \circ \operatorname{sh}(k) & -\operatorname{sh}(k) \circ \overline{\operatorname{sh}(k)}+\overline{\operatorname{ch}(k)} \circ \overline{\operatorname{ch}(k)}
\end{array}\right)
$$

we have $\overline{\operatorname{ch}(2 k)}=2 \operatorname{sh}(k) \circ \overline{\operatorname{sh}(k)}+1=2 \overline{\operatorname{ch}(k)} \circ \overline{\operatorname{ch}(k)}-1$ and $\operatorname{sh}(2 k)=$ $\overline{2 \operatorname{ch}(k)} \circ \operatorname{sh}(k)=2 \operatorname{sh}(k) \circ \operatorname{ch}(k)$. Using the above identities we can readily derive the identities,

$$
\begin{aligned}
& \mathbf{W}(\operatorname{sh}(k) \circ \overline{\operatorname{sh}(k)})=\mathbf{S}(\operatorname{sh}(k)) \circ \overline{\operatorname{sh}(k)}-\operatorname{sh}(k) \circ \overline{\mathbf{S}(\operatorname{sh}(k))} \\
& =\mathbf{W}(\overline{\operatorname{ch}(k)} \circ \overline{\operatorname{ch}(k)})=\mathbf{W}(\overline{\operatorname{ch}(k)}) \circ \overline{\operatorname{ch}(k)}+\overline{\operatorname{ch}(k)} \circ \mathbf{W}(\overline{\operatorname{ch}(k)}) \\
& \mathbf{S}(\overline{\operatorname{ch}(k)} \circ \operatorname{sh}(k))=\mathbf{W}(\overline{\operatorname{ch}(k)}) \circ \operatorname{sh}(k)+\overline{\operatorname{ch}(k)} \circ \mathbf{S}(\operatorname{sh}(k)) \\
& =\mathbf{S}(\operatorname{sh}(k) \circ \operatorname{ch}(k))=\mathbf{S}(\operatorname{sh}(k)) \circ \operatorname{ch}(k)-\operatorname{sh}(k) \circ \overline{\mathbf{W}(\overline{\operatorname{ch}(k)})} .
\end{aligned}
$$

The equations above have a generic character when we consider for example $\mathbf{S}(\bar{f} \circ g)=\mathbf{W}(\bar{f}) \circ g+\bar{f} \circ \mathbf{S}(g)$ etc.

Our original evolution equation, see [21] was

$$
\begin{aligned}
& \left(\frac{1}{i} \operatorname{sh}(k)_{t}+g^{T} \circ \operatorname{sh}(k)+\operatorname{sh}(k) \circ g-\overline{\operatorname{ch}(k)} \circ m\right) \circ \operatorname{ch}(k)= \\
& \left(\frac{1}{i} \overline{\operatorname{ch}(k)_{t}}+\left[g^{T}, \overline{\operatorname{ch}(k)}\right]+\operatorname{sh}(k) \circ \bar{m}\right) \circ \operatorname{sh}(k),
\end{aligned}
$$


or its equivalent form

$$
\begin{aligned}
& \frac{1}{i} \operatorname{sh}(k)_{t}+g^{T} \circ \operatorname{sh}(k)+\operatorname{sh}(k) \circ g-\overline{\operatorname{ch}(k)} \circ m \\
& -\left(\frac{1}{i} \overline{\operatorname{ch}(k)_{t}}+\left[g^{T}, \overline{\operatorname{ch}(k)}\right]+\operatorname{sh}(k) \circ \bar{m}\right) \circ(\overline{\operatorname{ch}(k)})^{-1} \circ \operatorname{sh}(k)=0 .
\end{aligned}
$$

The second equation can be written,

$\mathbf{S}(\operatorname{sh}(k))-\mathbf{W}(\overline{\operatorname{ch}(k)}) \circ(\overline{\operatorname{ch}(k)})^{-1} \circ \operatorname{sh}(k)=\overline{\operatorname{ch}(k)} \circ m+\operatorname{sh}(k) \circ \bar{m} \circ(\overline{\operatorname{ch}(k)})^{-1} \operatorname{sh}(k)$.

Multiplying on the left (86) with $(\overline{\operatorname{ch}(k)})^{-1}$ we obtain

$$
\begin{aligned}
& (\overline{\operatorname{ch}(k)})^{-1} \circ \mathbf{S}(\operatorname{sh}(k))-(\overline{\operatorname{ch}(k)})^{-1} \circ \mathbf{W}(\overline{\operatorname{ch}(k)}) \circ(\overline{\operatorname{ch}(k)})^{-1} \circ \operatorname{sh}(k)= \\
& m+(\overline{\operatorname{ch}(k)})^{-1} \operatorname{sh}(k) \circ \bar{m} \circ(\overline{\operatorname{ch}(k)})^{-1} \circ \operatorname{sh}(k)
\end{aligned}
$$

and using the fact that

$$
(\overline{\operatorname{ch}(k)})^{-1} \mathbf{W}(\overline{\operatorname{ch}(k)})(\overline{\operatorname{ch}(k)})^{-1}=-\mathbf{W}\left((\overline{\operatorname{ch}(k)})^{-1}\right)
$$

we have in view of the identities (84) the simple equation,

$$
\mathbf{S}(\zeta)=m+\zeta \circ \bar{m} \circ \zeta
$$

where $\zeta:=(\overline{\operatorname{ch}(k)})^{-1} \circ \operatorname{sh}(k)=\operatorname{sh}(k) \circ(\operatorname{ch}(k))^{-1}$. Now from (87) we can readily derive the equation below,

$$
\mathbf{W}(\operatorname{sh}(k) \circ \overline{\operatorname{sh}(k)})=m \circ \overline{\operatorname{sh}(k)} \circ \overline{\operatorname{ch}(k)}-\operatorname{sh}(k) \circ \operatorname{ch}(k) \circ \bar{m}
$$

which is the same as (43b) and it already appeared in [21]. To derive an equation for $\operatorname{sh}(2 k)$ notice that we can write,

$$
\operatorname{sh}(2 k)=(\overline{\operatorname{ch}(k)})^{2} \zeta+\zeta(\operatorname{ch}(k))^{2}
$$

and subsequently compute using (84), (87) and (88) in a tedious but straightforward manner,

$$
\begin{aligned}
\mathbf{S}(\operatorname{sh}(2 k)) & =\mathbf{W}\left(\overline{(\overline{\operatorname{ch}(k)})^{2}}\right) \zeta+(\overline{\operatorname{ch}(k)})^{2} \mathbf{S}(\zeta)+\mathbf{S}(\zeta)(\operatorname{ch}(k))^{2}-\zeta \overline{\left.\mathbf{W}(\overline{(\operatorname{ch}(k)})^{2}\right)} \\
& =[m \circ \overline{\operatorname{sh}(k)} \circ \overline{\operatorname{ch}(k)}-\operatorname{sh}(k) \circ \operatorname{ch}(k) \circ \bar{m}] \circ \overline{(\operatorname{ch}(k)})^{-1} \circ \operatorname{sh}(k) \\
& +(\overline{\operatorname{ch}(k)})^{2} \circ m+(\overline{\operatorname{ch}(k)})^{2} \circ(\overline{\operatorname{ch}(k)})^{-1} \circ \operatorname{sh}(k) \circ \bar{m} \circ(\overline{\operatorname{ch}(k)})^{-1} \operatorname{sh}(k) \\
& +m \circ(\operatorname{ch}(k))^{2}+\operatorname{sh}(k) \circ(\operatorname{ch}(k))^{-1} \circ \bar{m} \circ \operatorname{sh}(k) \circ(\operatorname{ch}(k))^{-1} \circ(\operatorname{ch}(k))^{2} \\
& -\operatorname{sh}(k) \circ(\operatorname{ch}(k))^{-1} \circ[\bar{m} \circ \operatorname{sh}(k) \circ \operatorname{ch}(k)-\operatorname{ch}(k) \circ \overline{\operatorname{sh}(k)} \circ m] \\
& =m \circ \operatorname{ch}(2 k)+\overline{\operatorname{ch}(2 k)} \circ m .
\end{aligned}
$$

This equation is (43a). 


\section{REFERENCES}

[1] Anderson, M. H., Ensher, J. R., Matthews, M. R., Wieman, C. E., Cornell, E.A.: Observation of Bose-Einstein condensation in a dilute atomic vapor. Science 269, 198-201 (1995)

[2] Bogoliubov, N. N.:Lectures on Quantum Statistics: Quasi-Averages, Gordon and Breach (1970)

[3] Bourgain, J. : Scattering in the energy space and below for 3d NLS, Journal d'analyse Mathématiques, 75, 267-297, (1998)

[4] Colliander, J, Keel, M., Staffilani, G, Takaoka, H, Tao, T. : Existence globale et diffusion pour l'équation de Schrödinger nonlinéaire répulsive cubique sur $\mathbb{R}^{3}$ en dessous l'espace d'énergie, Journees Equations aux derivees partielles Forges-les-Eaux, 3-7 juin 2002 GDR 2434 (CNRS)

[5] Chen, T. and Pavlović, N. : Derivation of the cubic NLS and Gross-Pitaevskii hierarchy for manybody dynamics in $d=2,3$ based on spacetime estimates., preprint.

[6] Chen, X. : Second order corrections to mean field evolution for weakly interacting bosons in the case of 3 body interactions, Arch. Rational Mech. Anal. 203 (2012), 455-497

[7] Chen, X. : Collapsing estimates and the rigorous derivation of the $2 \mathrm{~d}$ cubic nonlinear Schrödinger equation with anisotropic switchable quadratic traps, accepted, Journal de Mathématiques pures et appliquëes

[8] Chen, X. : On the rigorous derivation of the 3d cubic nonlinear Schrödinger equation with switchable quadratic traps, preprint

[9] Davis, K. B., Mewes, M.-O., Andrews, M. R., van Druten, N. J., Durfee, D. S., Kurn, D. M., Ketterle, W.: Bose-Einstein condensation in a gas of sodium atoms. Phys. Rev. Lett. 75, 3969-3973 (1995)

[10] Dyson, F.J.: Ground-state energy of a hard sphere gas. Phys. Rev. 106, 20-26 (1957)

[11] Elgart, A., Erdös, L., Schlein, B., Yau, H. T.: Gross-Pitaevskii equation as the mean field limit of weakly coupled bosons. Arch. Rat. Mech. Anal. 179, 265-283 (2006)

[12] Erdös, L., Yau, H. T.: Derivation of the non-linear Schrödinger equation from a many-body Coulomb system. Adv. Theor. Math. Phys. 5, 1169-1205 (2001)

[13] Erdös, L., Schlein, B., Yau, H. T.: Derivation of the Gross-Pitaevskii hierarchy for the dynamics of Bose-Einstein condensate. Comm. Pure Appl. Math. 59, 1659-1741 (2006)

[14] Erdös, L., Schlein, B., Yau, H. T.: Derivation of the cubic non-linear Schrödinger equation from quantum dynamics of many-body systems. Invent. Math. 167, 515-614 (2007)

[15] Erdös, L., Schlein, B., Yau, H. T.: Rigorous derivation of the Gross-Pitaevskii equation. Phys. Rev. Lett. 98, 040404 (2007)

[16] Erdös, L., Schlein, B., Yau, H. T.: Derivation of the Gross-Pitaevskii equation for the dynamics of Bose-Einstein condensate. Annals Math. 172, 291-370 (2010)

[17] Folland, G. B.: Harmonic analysis in phase space. Annals of Math. Studies, Vol. 122. Princeton, NJ: Princeton Univerity Press, 1989 
[18] Ginibre, J., Velo, G.: The classical field limit of scattering theory for nonrelativistic many-boson systems, I and II. Comm. Math. Phys. 66, 37-76 (1979) and 68, 45-68 (1979)

[19] Ginibre, J., Velo, G.: Scattering theory in the energy space for a class of nonlinear Schrödinger equations, J. Math. Pures Appl. 64 (1985), 363-401

[20] Grillakis, M. Machedon. M, Margetis, D.: Second-order corrections to mean field evolution of weakly interacting Bosons. I. Comm. Math. Phys. 294, 273301 (2010)

[21] Grillakis, M. Machedon. M, Margetis, D.: Second-order corrections to mean field evolution of weakly interacting Bosons. II. Adv. in Math. 228, 1788-1815 (2011)

[22] Gross, E. P.: Structure of a quantized vortex in boson systems. Nuovo Cim. 20, 454-477 (1961)

[23] Gross, E. P.: Hydrodynamics of a superfluid condensate. J. Math. Phys. 4, 195-207 (1963)

[24] Hepp, K.: The classical limit for quantum mechanical correlation functions. Comm. Math. Phys. 35, 265-277 (1974)

[25] Klainerman, S., Machedon,M. On the uniqueness of solutions to the GrossPitaevskii hierarchy. Comm. Math. Phys. 279, 169-185 (2008)

[26] Kirkpatrick, K., Schlein, B., Staffilani, G. :Derivation of the two dimensional nonlinear Schrödinger equation from manybody quantum dinamics, Amer. J. Math, 133 (2011), 91-130.

[27] Lee, T. D., Huang, K., Yang, C. N.: Eigenvalues and eigenfunctions of a Bose system of hard spheres and its low-temperature properties. Phys. Rev. 106, 1135-1145 (1957)

[28] Lee, T. Yang, C. N.: Low-Temperature Behaviour of a Dilute System of Hard Spheres. I. Equilibrium Properties. Phys. Rev. 112, 1419-1429 (1958)

[29] Lieb, E. H., Seiringer, R., Solovej, J. P., Yngvanson, J.: The mathematics of the Bose gas and its condensation. Basel, Switzerland: Birkhaüser Verlag, 2005

[30] Lin, J, Strauss, W. Decay and scattering of solutions of a nonlinear Schrödinger equation, Journ. Funct. Anal. 30, (1978), 245-263.

[31] Margetis, D.: Solvable model for pair excitation in trapped Boson gas at zero temperature. J. Phys. A: Math. Theor. 41, 235004 (2008); Corrigendum. J. Phys. A: Math. Theor. 41, 459801 (2008)

[32] Pitaevskii, L. P.: Vortex lines in an imperfect Bose gas. Soviet Phys. JETP 13, 451-454 (1961)

[33] Pitaevskii, L. P., Stringari, S.: Bose-Einstein condensation. Oxford, UK: Oxford University Press, 2003

[34] Riesz, F., Nagy, B.: Functional analysis. New York: Frederick Ungar Publishing, 1955

[35] Rodnianski, I., Schlein, B.: Quantum fluctuations and rate of convergence towards mean field dynamics. Comm. Math. Phys. 291(2), 31-61 (2009)

[36] Shale, D.: Linear symmetries of free Boson fields, Trans. Amer. Math. Soc. 103(1), 149-167 (1962)

[37] $\mathrm{Wu}, \mathrm{T}$. T.: Some nonequilibrium properties of a Bose system of hard spheres at extremely low temperatures. J. Math. Phys. 2, 105-123 (1961) 
[38] $\mathrm{Wu}, \mathrm{T}$. T.: Bose-Einstein condensation in an external potential at zero temperature: General Theory. Phys. Rev. A 58, 1465-1474 (1998)

University of Maryland, College Park

E-mail address: mng@math.umd.edu

University of Maryland, College Park

E-mail address: mxm@math.umd.edu 